\title{
EXISTENCE AND NONEXISTENCE OF POSITIVE SOLUTIONS TO SOME FULLY NONLINEAR EQUATION IN ONE DIMENSION
}

\author{
PATRICIO FELMER AND NORIHISA IKOMA
}

\begin{abstract}
In this paper, we consider the existence (and nonexistence) of solutions to$$
-\mathcal{M}_{\lambda, \Lambda}^{ \pm}\left(u^{\prime \prime}\right)+V(x) u=f(u) \quad \text { in } \mathbf{R}
$$

where $\mathcal{M}_{\lambda, \Lambda}^{+}$and $\mathcal{M}_{\lambda, \Lambda}^{-}$denote the Pucci operators with $0<\lambda \leq \Lambda<$ $\infty, V(x)$ is a bounded function, $f(s)$ is a continuous function and its typical example is a power-type nonlinearity $f(s)=|s|^{p-1} s(p>1)$. In particular, we are interested in positive solutions which decay at infinity, and the existence (and nonexistence) of such solutions is proved.
\end{abstract}

\section{INTRODUCTION}

In this paper, we study the existence and nonexistence of solutions to the following nonlinear differential equations

$$
-\mathcal{M}_{\lambda, \Lambda}^{ \pm}\left(u^{\prime \prime}\right)+V(x) u=f(u) \quad \text { in } \mathbf{R}, \quad u>0 \quad \text { in } \mathbf{R}, \quad \lim _{|x| \rightarrow \infty} u(x)=0 .
$$

Here $V$ and $f$ are given functions, $0<\lambda \leq \Lambda<\infty$ constants and $\mathcal{M}_{\lambda, \Lambda}^{ \pm}(s)$ the Pucci operators defined by

$$
\mathcal{M}_{\lambda, \Lambda}^{+}(s):=\left\{\begin{array}{ll}
\Lambda s & \text { if } s \geq 0, \\
\lambda s & \text { if } s<0,
\end{array} \quad \mathcal{M}_{\lambda, \Lambda}^{-}(s):= \begin{cases}\lambda s & \text { if } s \geq 0 \\
\Lambda s & \text { if } s<0\end{cases}\right.
$$

We remark that when $\lambda=\Lambda$, one has $\mathcal{M}_{\lambda, \Lambda}^{ \pm}\left(u^{\prime \prime}\right)=\lambda u^{\prime \prime}$.

One of motivations to study equations like (1.1) is to see to what extent the properties and the results in the semilinear case can be generalized to the fully nonlinear case. When $\lambda=\Lambda,(1.1)$ is well studied and it is proved that (1.1) has a solution for various $V(x)$ and $f(s)$ by critical point theory. Here we refer to $[10,11]$ and references therein.

On the other hand, when $\lambda \neq \Lambda,(1.1)$ is not studied well. In [7], instead of (1.1), the authors study the existence of positive radial solutions of

$$
-\mathcal{M}_{\lambda, \Lambda}^{ \pm}\left(D^{2} u\right)+\gamma u=f(u) \quad \text { in } B_{R}(0) \subset \mathbf{R}^{N}, \quad u=0 \quad \text { on } \partial B_{R}(0)
$$

as well as

$$
-\mathcal{M}_{\lambda, \Lambda}^{ \pm}\left(D^{2} u\right)+u=u^{p} \quad \text { in } \mathbf{R}^{N}
$$

Date: August 11, 2021. 
Here $N \geq 3,0 \leq \gamma$ and $1<p<p_{*}^{ \pm}$where $p_{*}^{ \pm}$are critical exponents for $\mathcal{M}_{\lambda, \Lambda}^{ \pm}$(see also $[1,3,5,6]$ ). Recently, in [8], the authors show the existence of infinitely many radial solutions of (1.2) when $\gamma=0$ and $f(s)=|s|^{p-1} s$. Moreover, in [8], the inhomogeneous case is also considered and the existence of infinitely many solutions is shown on a bounded annulus.

In this paper, we aim to treat the inhomogeneous equation on the unbounded domain $\mathbf{R}$. We emphasis that in general the existence of solutions to (1.1) is delicate when the equation is inhomogeneous and the domain is unbounded. Indeed, we shall prove the nonexistence result when $V(x)$ is monotone. See Theorem 1.2 below.

We first deal with the existence result. For $V(x)$, we assume

(V1) $V \in W^{1, \infty}(\mathbf{R})$ and $0<\inf _{\mathbf{R}} V=: V_{0}$.

(V2) For a.a. $x \in(-\infty, 0)$ and a.a. $y \in(0, \infty), V^{\prime}(x) \leq 0 \leq V^{\prime}(y)$.

(V3) $V(0) \leq V_{\infty}:=\lim _{|x| \rightarrow \infty} V(x)$ and there exist $C_{0}, \xi_{0}>0$ such that

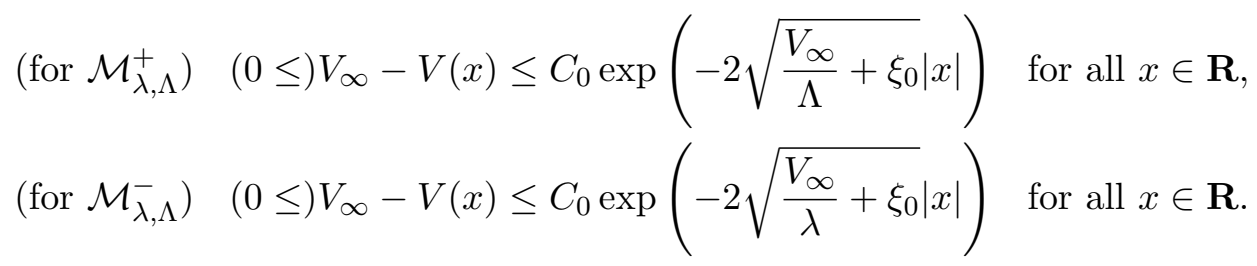

Next, for $f(s)$, we suppose the following conditions and an example of $f(s)$ is $f(s)=\sum_{i=1}^{k} a_{i} s^{p_{i}}$ where $0<a_{i}$ and $1<p_{i}$ :

(f1) $f \in C^{1}(\mathbf{R})$ and $f(s)=0$ for all $s \leq 0$.

(f2) There exists an $\eta_{0}>0$ such that $\lim _{s \rightarrow 0} s^{-1-\eta_{0}} f(s)=0$.

(f3) As $s \rightarrow \infty$,

$$
\frac{f(s)}{s} \rightarrow \infty \quad \text { and } \quad \frac{f(\theta s)}{f(s)} \rightarrow \bar{f}(\theta) \quad \text { in } C_{\mathrm{loc}}((0,1]) .
$$

(f4) $s \mapsto s^{-1} f(s):(0, \infty) \rightarrow \mathbf{R}$ is strictly increasing.

Remark 1.1 (i) In (f3), it follows that $\bar{f} \in C((0,1]), \bar{f}(1)=1$ and $\bar{f}(\theta) \geq 0$ for $\theta \in(0,1]$. For example, when $f(s)=s^{p}$ and $f(s)=s \log s$, one sees $\bar{f}(\theta)=\theta^{p}$ and $\bar{f}(\theta)=\theta$ respectively.

(ii) When $\lambda=\Lambda$, condition ( $\left.f_{4}\right)$ is used to obtain bounded Palais-Smale sequences. The classical condition to obtain bounded Palais-Smale sequences is the Ambrosetti-Rabinowitz condition: $0<\mu \int_{0}^{s} f(t) \mathrm{d} t \leq f(s)$ s for some $\mu>2$ and all $s>0$. We remark that $(f 1)-(f 4)$ do not imply this condition. In fact, consider a function defined by

$$
f(s)=\eta(s) s^{p}+(1-\eta(s)) C s \log s
$$

where $1<p, \eta \in C^{\infty}([0, \infty), \mathbf{R}), \eta^{\prime}(s) \leq 0$ for every $s \in[0, \infty), \eta(s)=1$ if $0 \leq s \leq 2, \eta(s)=0$ if $3 \leq s$ and $C>0$ is chosen so that $C \log s \geq s^{p-1}$ in $[2,3]$. It is easily seen that $f$ satisfies $(f 1)-\left(f_{4}\right)$ with $\bar{f}(\theta)=\theta$ and that $F(s)$ has the growth $s^{2} \log (s)$ as $s \rightarrow \infty$, providing the required counterexample. 
Under these conditions, we have

Theorem 1.1. Under (V1)-(V3) and (f1)-(f4), (1.1) have a solution.

Next, we turn to the nonexistence result. In this case, we assume that $V(x)$ is monotone:

(V2') $V^{\prime}(x) \geq 0$ in $\mathbf{R}$ and

$$
\underline{V}=\lim _{x \rightarrow-\infty} V(x)<\lim _{x \rightarrow \infty} V(x)=\bar{V} .
$$

Then we have

Theorem 1.2. Let $0<\lambda \leq \Lambda<\infty$ and assume (V1), (V2'), (f1), (f4) and

$$
\lim _{s \rightarrow 0} \frac{f(s)}{s}=0 .
$$

Then (1.1) have no solution.

Remark 1.2 Theorem 1.2 still holds when we replace (V2') by

$$
V^{\prime}(x) \leq 0 \quad \text { in } \mathbf{R}, \quad \bar{V}=\lim _{x \rightarrow-\infty}>\lim _{x \rightarrow \infty} V(x)=\underline{V} .
$$

Here we make some comments on the proofs of Theorems 1.1 and 1.2. First, even though equation (1.1) can be transformed into an equation with variational structure (pointed by Professor Evans), we prefer to use degree theoretic arguments in view of future applications. For Theorem 1.1, we borrow the idea in [4] (cf. [7]). More precisely, we will find a suitable function space $X$ which is a Banach space, and rewrite (1.1) into the equations (id $\left.\mathcal{L}^{ \pm}\right)(u)=0$ where $\mathcal{L}^{ \pm}(u):=\left(-\mathcal{M}_{\lambda, \Lambda}^{ \pm}+V(x)\right)^{-1} f(u(x))$ for $u \in X$. To find a solution $u \neq 0$, we use the Leray-Schauder $\operatorname{degree} \operatorname{deg}_{X}$ in $X$ and prove that

i) There exists an $r_{0}>0$ such that $\operatorname{deg}_{X}\left(\right.$ id $\left.-\mathcal{L}^{ \pm}, B_{r_{0}}(0), 0\right)=1$.

ii) There exists an $r_{1}>r_{0}$ such that $\operatorname{deg}_{X}\left(\mathrm{id}-\mathcal{L}^{ \pm}, B_{r_{1}}(0), 0\right)=0$. From i) and ii), we have $\operatorname{deg}_{X}\left(\mathrm{id}-\mathcal{L}^{ \pm}, A_{r_{0}, r_{1}}, 0\right) \neq 0$ and find a $u_{0} \in A_{r_{1}, r_{2}}$ so that $\left(\mathrm{id}-\mathcal{L}^{ \pm}\right)\left(u_{0}\right)=0$ where $A_{r_{1}, r_{2}}:=\left\{u \in X \mid r_{1}<\|u\|_{X}<r_{2}\right\}$. One of difficulties here is to find a suitable $X$ in order that we can prove the property ii) as well as the map $\mathcal{L}^{ \pm}: X \rightarrow X$ is compact. A key for proving ii) is a priori estimates of solutions in $X$. Since we treat the unbounded domain, we need the uniform decay estimates of solutions as well as the uniform $L^{\infty}$-bounds. This point is different from the bounded domain case and requires delicate arguments. For instance, see Proposition 2.9 below.

We also point out that the argument of Proposition 2.9 is useful to show the nonexistence result namely, Theorem 1.2. Indeed, this case is simpler than Proposition 2.9 and we will prove Theorem 1.2 in section 3.

In Appendix A, we consider (1.1) in the special case when $V(x) \equiv$ const. > 0 . In this case, we can prove the unique existence of solutions up to translations. See Proposition 2.1 and Appendix A. 


\section{Proof of Theorem 1.1}

Throughout this section, we always assume (f1)-(f4) and (V1)-(V3). We begin with the existence result when $V(x) \equiv$ const. $>0$.

Proposition 2.1. Under (f1)-(f4), the equations

$$
\left\{\begin{array}{l}
-\mathcal{M}_{\lambda, \Lambda}^{+}\left(u^{\prime \prime}\right)+V_{\infty} u=f(u) \quad \text { in } \mathbf{R}, \quad u>0 \text { in } \mathbf{R}, \\
u(x) \rightarrow 0 \text { as }|x| \rightarrow \infty, \quad u(0)=\max _{x \in \mathbf{R}} u(x)
\end{array}\right.
$$

and

$$
\left\{\begin{array}{l}
-\mathcal{M}_{\lambda, \Lambda}^{-}\left(u^{\prime \prime}\right)+V_{\infty} u=f(u) \quad \text { in } \mathbf{R}, \quad u>0 \text { in } \mathbf{R}, \\
u(x) \rightarrow 0 \text { as }|x| \rightarrow \infty, \quad u(0)=\max _{x \in \mathbf{R}} u(x)
\end{array}\right.
$$

have unique solutions $\omega_{+}$and $\omega_{-}$. Furthermore, there exist $z^{ \pm}>0, c_{1}>0$ and $c_{2}>0$ such that

$$
\begin{array}{ll}
\omega_{ \pm}^{\prime \prime}(x)<0=\omega_{ \pm}^{\prime \prime}\left(z^{ \pm}\right)<\omega_{ \pm}^{\prime \prime}(y) & \text { for every } x, y \in \mathbf{R} \text { with }|x|<z^{ \pm}<|y|, \\
\omega_{ \pm}(x) \leq c_{1} \exp \left(-c_{2}|x|\right) & \text { for all } x \in \mathbf{R} .
\end{array}
$$

Finally, if u satisfies

$$
\begin{aligned}
& \mathcal{M}_{\lambda, \Lambda}^{ \pm}\left(u^{\prime \prime}\right)+V_{\infty} u=f(u) \text { in } \mathbf{R}, \quad u>0 \text { in } \mathbf{R}, \\
& u(0)=\max _{\mathbf{R}} u, \quad u(x) \rightarrow 0 \text { if } x \rightarrow \infty \text { or } x \rightarrow-\infty,
\end{aligned}
$$

then $u=\omega_{ \pm}$.

We shall prove Proposition 2.1 in Appendix A.

From now on, we may assume $V(0)<V_{\infty}$ in (V3) and $V(x)$ is not a constant function without loss of generality. Under this additional assumption, we fix an $\eta_{1}>0$ so that

$$
\eta_{1}<c_{2}, \quad \Lambda \eta_{1}^{2}\left(1+\frac{\eta_{0}}{2}\right)^{2}<\frac{V_{0}}{2}
$$

where $V_{0}:=\inf _{\mathbf{R}} V>0$, and $\eta_{0}>0$ and $c_{2}>0$ appear in (f2) and Proposition 2.1. We set

$$
X_{\eta_{1}}:=\left\{v \in C(\mathbf{R})\left|\|v\|_{\eta_{1}}=\sup _{x \in \mathbf{R}} e^{\eta_{1}|x|}\right| v(x) \mid<\infty\right\} .
$$

It is easy to check that $\left(X_{\eta_{1}},\|\cdot\|_{\eta_{1}}\right)$ is a Banach space.

Lemma 2.2. For every $v \in X_{\eta_{1}}$, the equations

$$
-\mathcal{M}_{\lambda, \Lambda}^{ \pm}\left(u^{\prime \prime}\right)+V(x) u=f(v(x)) \quad \text { in } \mathbf{R}, \quad u \in X_{\eta_{1}},
$$

have unique solutions.

Proof. We prove the claim at the same time for $\mathcal{M}_{\lambda, \Lambda}^{+}$and $\mathcal{M}_{\lambda, \Lambda}^{-}$. Let $v \in$ $X_{\eta_{1}}$. For each $n \in \mathbf{N}$, consider

$$
\left\{\begin{aligned}
-\mathcal{M}_{\lambda, \Lambda}^{ \pm}\left(u^{\prime \prime}\right)+V(x) u & =f(v(x)) \quad \text { in }(-n, n), \\
u(-n)=0 & =u(n) .
\end{aligned}\right.
$$


Then the above equations have a unique solution $u_{n} \in C^{2}([-n, n])$ due to (V1). In fact, since $f(s) \geq 0$ by (f1), (f2) and (f4), $u \equiv 0$ is a subsolution of the above equation. In addition, one can check that the principal eigenvalues of $-\mathcal{M}_{\lambda, \Lambda}^{ \pm}+V(x)$ on $[-n, n]$ with the Dirichlet zero boundary condition are positive due to (V1). Thus, by (f2), the positive eigenfunctions multiplied by small positive constants become supersolutions and so, the solution $u_{n}$ is unique.

Now, the maximum principle yields $u_{n} \geq 0$ in $[-n, n]$. Moreover, we have

$$
\left\|u_{n}\right\|_{L^{\infty}(-n, n)} \leq V_{0}^{-1}\|f(v)\|_{L^{\infty}(\mathbf{R})} .
$$

Indeed, let $x_{n} \in(-n, n)$ be a maximum point of $u_{n}$. It follows from the equation and $u_{n}^{\prime \prime}\left(x_{n}\right) \leq 0$ that

$$
\left\|u_{n}\right\|_{L^{\infty}(-n, n)}=u_{n}\left(x_{n}\right) \leq \frac{\|f(v)\|_{L^{\infty}(\mathbf{R})}}{V\left(x_{n}\right)} \leq \frac{\|f(v)\|_{L^{\infty}(\mathbf{R})}}{V_{0}} .
$$

Next we shall show that there exist $C_{3}>0$ and $\delta_{0}>\eta_{1}$ such that

$$
u_{n}(x) \leq C_{3} e^{-\delta_{0}|x|} \quad \text { for all } x \in \mathbf{R} \text { and } n \geq 1 .
$$

To this end, we first notice that (f2) yields

$$
f(s) \leq C_{4}|s|^{1+\eta_{0}} \quad \text { for all }|s| \leq\|v\|_{L^{\infty}} .
$$

Hence, by the definition of $\|\cdot\|_{\eta_{1}}$, we obtain

$$
f(v(x)) \leq C_{4}|v(x)|^{1+\eta_{0}} \leq C_{4}\|v\|_{\eta_{1}}^{1+\eta_{0}} e^{-\left(1+\eta_{0}\right) \eta_{1}|x|} \leq C_{5} e^{-\left(1+\eta_{0}\right) \eta_{1}|x|}
$$

for all $x \in \mathbf{R}$. Recalling (2.3), fix an $R_{0}>0$ so that

$$
-\Lambda \eta_{1}^{2}\left(1+\frac{\eta_{0}}{2}\right)^{2}+V_{0}-C_{5} e^{-\eta_{0} \eta_{1} R_{0} / 2} \geq \frac{V_{0}}{4}>0 .
$$

We only treat $n$ with $n>R_{0}$ and set

$$
M:=1+\frac{\|f(v)\|_{L^{\infty}}}{V_{0}}, \quad \omega_{0}(x):=M e^{-\left(1+\frac{\eta_{0}}{2}\right) \eta_{1}\left(|x|-R_{0}\right)}
$$

Noting (2.3), (2.6), (2.7) and

$$
\omega_{0}^{\prime \prime}=\left(1+\frac{\eta_{0}}{2}\right)^{2} \eta_{1}^{2} \omega_{0} \geq 0, \quad M \geq 1
$$


we get the following: for all $R_{0} \leq|x| \leq n$,

$$
\begin{aligned}
& -\mathcal{M}_{\lambda, \Lambda}^{ \pm}\left(\omega_{0}^{\prime \prime}\right)+V \omega_{0}-f(v) \\
\geq & -\mathcal{M}_{\lambda, \Lambda}^{ \pm}\left(\omega_{0}^{\prime \prime}\right)+V \omega_{0}-M f(v) \\
\geq & \left\{-\Lambda\left(1+\frac{\eta_{0}}{2}\right)^{2} \eta_{1}^{2}+V\right\} \omega_{0}-M C_{5} e^{-\left(1+\eta_{0}\right) \eta_{1}|x|} \\
\geq & {\left[\left\{-\Lambda\left(1+\frac{\eta_{0}}{2}\right)^{2} \eta_{1}^{2}+V\right\} e^{\left(1+\frac{\eta_{0}}{2}\right) \eta_{1} R_{0}}-C_{5} e^{-\eta_{0} \eta_{1}|x| / 2}\right] M e^{-\left(1+\frac{\eta_{0}}{2}\right) \eta_{1}|x|} } \\
\geq & {\left[-\Lambda\left(1+\frac{\eta_{0}}{2}\right)^{2} \eta_{1}^{2}+V_{0}-C_{5} e^{-\eta_{0} \eta_{1} R_{0} / 2}\right] M e^{-\left(1+\frac{\eta_{0}}{2}\right) \eta_{1}|x|} } \\
\geq & 0 .
\end{aligned}
$$

Since

$$
\begin{aligned}
& \mathcal{M}_{\lambda, \Lambda}^{+}\left(m_{1}\right)-\mathcal{M}_{\lambda, \Lambda}^{+}\left(m_{2}\right) \geq \mathcal{M}_{\lambda, \Lambda}^{-}\left(m_{1}-m_{2}\right), \\
& \mathcal{M}_{\lambda, \Lambda}^{-}\left(m_{1}\right)-\mathcal{M}_{\lambda, \Lambda}^{-}\left(m_{2}\right) \geq \mathcal{M}_{\lambda, \Lambda}^{-}\left(m_{1}-m_{2}\right)
\end{aligned}
$$

for all $m_{1}, m_{2} \in \mathbf{R}$, we have

$$
-\mathcal{M}_{\lambda, \Lambda}^{-}\left(\omega_{0}^{\prime \prime}-u_{n}^{\prime \prime}\right)+V(x)\left(\omega_{0}-u_{n}\right) \geq 0
$$

for each $R_{0} \leq|x| \leq n$. From (2.4) and the definitions of $M$ and $\omega_{0}$, we have

$$
u_{n}\left( \pm R_{0}\right) \leq M=\omega_{0}\left( \pm R_{0}\right), \quad 0=u_{n}( \pm n)<\omega_{0}( \pm n) .
$$

By the comparison principle, we get

$$
u_{n}(x) \leq \omega_{0}(x) \text { for all } R_{0} \leq|x| \leq n .
$$

Thus, (2.5) holds with $\delta_{0}:=\left(1+\eta_{0} / 2\right) \eta_{1}$.

By the elliptic regularity, one sees that $\left(u_{n}\right)$ is bounded in $C_{\text {loc }}^{2}(\mathbf{R})$, hence there exists $\left(u_{n_{k}}\right)$ such that $u_{n_{k}} \rightarrow u_{0}$ in $C_{\text {loc }}^{2}(\mathbf{R})$, where $u_{0}$ satisfies

$$
-\mathcal{M}_{\lambda, \Lambda}^{ \pm}\left(u_{0}^{\prime \prime}\right)+V(x) u_{0}=f(v(x)) \text { in } \mathbf{R} \text {. }
$$

Moreover, from (2.5), we obtain

$$
u_{0}(x) \leq C_{6} e^{-\delta_{0}|x|} \quad \text { in } \mathbf{R} .
$$

Since $\delta_{0}>\eta_{1}, u_{0} \in X_{\eta_{1}}$ and the existence of solutions is proved.

For the uniqueness, let $u_{1}, u_{2} \in X_{\eta_{1}}$ be solutions of

$$
-\mathcal{M}_{\lambda, \Lambda}^{ \pm}\left(u^{\prime \prime}\right)+V(x) u=f(v(x)) \text { in } \mathbf{R}
$$

and set $w(x):=u_{1}(x)-u_{2}(x)$. Then it follows from (2.8) that $\pm w(x)$ satisfy

$$
-\mathcal{M}_{\lambda, \Lambda}^{-}\left(u^{\prime \prime}\right)+V(x) u \geq 0 \quad \text { in } \mathbf{R} .
$$

Noting that $w(x) \rightarrow 0$ as $|x| \rightarrow \infty$, combining with the above inequality, $\pm w(x)$ do not have any negative minimum on $\mathbf{R}$. Hence, $w \equiv 0$ and $u_{1} \equiv u_{2}$. Thus we complete the proof.

Definition 2.3. For $v \in X_{\eta_{1}}$, we denote by $\mathcal{L}^{ \pm}(v)$ the unique solutions of

$$
-\mathcal{M}_{\lambda, \Lambda}^{ \pm}\left(u^{\prime \prime}\right)+V(x) u=f(v) \quad \text { in } \mathbf{R}, \quad u \in X_{\eta_{1}} .
$$


Thanks to Lemma 2.2, $\mathcal{L}^{ \pm}: X_{\eta_{1}} \rightarrow X_{\eta_{1}}$. Furthermore,

Lemma 2.4. The maps $\mathcal{L}^{ \pm}: X_{\eta_{1}} \rightarrow X_{\eta_{1}}$ are compact.

Proof. Let $\left(v_{n}\right) \subset X_{\eta_{1}}$ be a bounded sequence and put $u_{n}=\mathcal{L}^{ \pm}\left(v_{n}\right)$. We first show that $\left(u_{n}\right)$ has a convergent subsequence in $X_{\eta_{1}}$. Set

$$
M_{1}=\sup _{n \geq 1}\left\|v_{n}\right\|_{\eta_{1}}
$$

Then we have

$$
v_{n}(x) \leq M_{1} e^{-\eta_{1}|x|} \text { for all } x \in \mathbf{R},
$$

and there exists an $M_{2}>0$ such that

$$
\left\|u_{n}\right\|_{L^{\infty}} \leq \frac{\left\|f\left(v_{n}\right)\right\|_{L^{\infty}}}{V_{0}} \leq M_{2} \text { for all } n \geq 1
$$

(see the beginning of proof of Lemma 2.2). Now as in (2.6) and (2.7), choose an $R_{2}>0$ so large that, for $|x| \geq R_{2}$ we have

$$
\left|f\left(v_{n}(x)\right)\right| \leq C_{7} e^{-\left(1+\eta_{0}\right) \eta_{1}|x|}
$$

and

For $R>R_{2}$, set

$$
-\Lambda \eta_{1}^{2}\left(1+\frac{\eta_{0}}{2}\right)^{2}+V_{0}-C_{7} e^{-\eta_{0} \eta_{1}|x| / 2} \geq \frac{V_{0}}{4}>0 .
$$

$$
w_{R}(x):=M_{3}\left[e^{-\left(1+\frac{\eta_{0}}{2}\right) \eta_{1}\left(|x|-R_{2}\right)}+e^{\left(1+\frac{\eta_{0}}{2}\right) \eta_{1}(|x|-R)}\right]
$$

where $M_{3}:=1+M_{2}$. Since $w_{R}^{\prime \prime}=\left(1+\frac{\eta_{0}}{2}\right)^{2} \eta_{1}^{2} w_{R} \geq 0$, as in the proof of Lemma 2.2, for all $R_{2} \leq|x| \leq R$, we get

$$
\begin{aligned}
& -\mathcal{M}_{\lambda, \Lambda}^{ \pm}\left(w_{R}^{\prime \prime}\right)+V(x) w_{R}-f\left(v_{n}\right) \\
\geq & -\mathcal{M}_{\lambda, \Lambda}^{ \pm}\left(w_{R}^{\prime \prime}\right)+V(x) w_{R}-M_{3} f\left(v_{n}\right) \\
\geq & \left\{-\Lambda\left(1+\frac{\eta_{0}}{2}\right)^{2} \eta_{1}^{2}+V\right\} M_{3}\left[e^{-\left(1+\frac{\eta_{0}}{2}\right) \eta_{1}\left(|x|-R_{2}\right)}+e^{\left(1+\frac{\eta_{0}}{2}\right) \eta_{1}(|x|-R)}\right] \\
& -M_{3} C_{7} e^{-\left(1+\eta_{0}\right) \eta_{1}|x|} \\
\geq & {\left[-\Lambda\left(1+\frac{\eta_{0}}{2}\right)^{2} \eta_{1}^{2}+V_{0}-C_{7} e^{-\eta_{0} \eta_{1} R_{2} / 2}\right] M_{3} e^{-\left(1+\frac{\eta_{0}}{2}\right) \eta_{1}|x|} } \\
& +\left[-\Lambda\left(1+\frac{\eta_{0}}{2}\right)^{2} \eta_{1}^{2}+V_{0}\right] e^{\left(1+\frac{\eta_{0}}{2}\right) \eta_{1}(|x|-R)} \\
\geq & 0=-\mathcal{M}_{\lambda, \Lambda}^{ \pm}\left(u_{n}^{\prime \prime}\right)+V(x) u_{n}-f\left(v_{n}\right) .
\end{aligned}
$$

Noting

$$
0 \leq u_{n}\left( \pm R_{2}\right) \leq M_{2} \leq w_{R}\left( \pm R_{2}\right) \quad \text { and } \quad 0 \leq u_{n}( \pm R) \leq M_{2} \leq w_{R}( \pm R),
$$

the comparison principle gives

$$
u_{n}(x) \leq w_{R}(x)=M_{3}\left[e^{-\left(1+\frac{\eta_{0}}{2}\right) \eta_{1}\left(|x|-R_{2}\right)}+e^{\left(1+\frac{\eta_{0}}{2}\right) \eta_{1}(|x|-R)}\right],
$$


for all $R_{2} \leq|x| \leq R$ and $n \geq 1$. Letting $R \rightarrow \infty$, we obtain

$$
u_{n}(x) \leq C_{8} e^{-\left(1+\frac{\eta_{0}}{2}\right) \eta_{1}|x|} \text { for all } x \in \mathbf{R} \text { and } n \geq 1 \text {. }
$$

Using this exponential decay and the equation, we observe that there exists $C_{9}>0$ such that

$$
\left\|u_{n}\right\|_{L^{\infty}}+\left\|u_{n}^{\prime}\right\|_{L^{\infty}}+\left\|u_{n}^{\prime \prime}\right\|_{L^{\infty}} \leq C_{9} \text { for all } n \geq 1 .
$$

Thus, there exists $\left(u_{n_{k}}\right)$ such that $u_{n_{k}} \rightarrow u_{0}$ in $C_{\text {loc }}^{2}(\mathbf{R})$, where $u_{0}$ satisfies

$$
u_{0}(x) \leq C_{8} e^{-\left(1+\frac{\eta_{0}}{2}\right) \eta_{1}|x|} \quad \text { for all } x \in \mathbf{R} .
$$

This implies that $u_{0} \in X_{\eta_{1}}$ and $u_{n_{k}} \rightarrow u_{0}$ in $X_{\eta_{1}}$. Hence, $\left(u_{n}\right)$ is relatively compact in $X_{\eta_{1}}$.

Finally, we prove the continuity of $\mathcal{L}^{ \pm}$. If $v_{n} \rightarrow v_{0}$ in $X_{\eta_{1}}$, then arguing as in the above, there exists a subsequence $\left(u_{n_{k}}\right)$ such that $u_{n_{k}} \rightarrow u_{0}$ in $X_{\eta_{1}} \cap C_{\text {loc }}^{2}(\mathbf{R})$ where $u_{0}$ satisfies

$$
-\mathcal{M}_{\lambda, \Lambda}^{ \pm}\left(u_{0}^{\prime \prime}\right)+V(x) u_{0}=f\left(v_{0}\right) \text { in } \mathbf{R} .
$$

By Lemma 2.2, $u_{0}$ is uniquely determined and does not depend on choices of subsequences. Therefore, it is easily seen that the whole sequence $\left(u_{n}\right)$ converges to $u_{0}$ in $X_{\eta_{1}}$ and the maps $\mathcal{L}^{ \pm}$are continuous.

Using $\mathcal{L}^{ \pm}$, the fact $f(s) \geq 0$ for every $s \in \mathbf{R}$ and the strong maximum principle, we notice that $u \in X_{\eta_{1}}$ is a solution of (1.1) if and only if $u=$ $\mathcal{L}^{ \pm}(u)$ with $u \neq 0$.

Next, in order to find a nontrivial fixed point of $\mathcal{L}^{ \pm}$in $X_{\eta_{1}}$, following the idea in [4] (cf. [7]), we shall show that

i) There exists an $r_{0}>0$ such that $\operatorname{deg}_{X_{\eta_{1}}}\left(\mathrm{id}-\mathcal{L}^{ \pm}, B_{r_{0}}(0), 0\right)=1$.

ii) There exists an $r_{1}>r_{0}$ such that $\operatorname{deg}_{X_{\eta_{1}}}\left(\mathrm{id}-\mathcal{L}^{ \pm}, B_{r_{1}}(0), 0\right)=0$.

Here $\operatorname{deg}_{X_{\eta_{1}}}\left(\mathrm{id}-\mathcal{L}^{ \pm}, \Omega, 0\right)$ stands for the degree of the map id $-\mathcal{L}^{ \pm}$in $X_{\eta_{1}}$. From i) and ii), it follows that

$$
\text { id }-\mathcal{L}^{ \pm} \neq 0 \quad \text { on } \partial A_{r_{0}, r_{1}} \quad \text { and } \quad \operatorname{deg}_{X_{\eta_{1}}}\left(\mathrm{id}-\mathcal{L}^{ \pm}, A_{r_{0}, r_{1}}, 0\right)=-1
$$

where $A_{r_{0}, r_{1}}:=\left\{u \in X_{\eta_{1}} \mid r_{0}<\|u\|_{\eta_{1}}<r_{1}\right\}$. Thus, if we can prove i) and ii) we can find a solution of (1.1) in $A_{r_{0}, r_{1}}$.

First we show i), namely,

Lemma 2.5. There exists an $r_{0}>0$ such that $\operatorname{deg}_{X_{\eta_{1}}}\left(\mathrm{id}-\mathcal{L}^{ \pm}, B_{r_{0}}(0), 0\right)=1$.

Proof. It suffices to prove that there exists an $r_{0}>0$ such that (id $\left.\beta \mathcal{L}^{ \pm}\right)(u) \neq 0$ for all $u \in \partial B_{r_{0}}(0)$ and all $\beta \in[0,1]$ since the homotopy invariance gives

$$
\operatorname{deg}_{X_{\eta_{1}}}\left(\mathrm{id}-\mathcal{L}^{ \pm}, B_{r_{0}}(0), 0\right)=\operatorname{deg}_{X_{\eta_{1}}}\left(\mathrm{id}, B_{r_{0}}(0), 0\right)=1 .
$$

We first notice that for $\beta>0$, the equations $u=\beta \mathcal{L}^{ \pm}(u)$ are equivalent to

$$
-\mathcal{M}_{\lambda, \Lambda}^{ \pm}\left(u^{\prime \prime}\right)+V(x) u=\beta f(u) \text { in } \mathbf{R}
$$


for $u \in X_{\eta_{1}}$. If $u \in X_{\eta_{1}} \backslash\{0\}$ satisfies $u=\beta \mathcal{L}^{ \pm}(u)$ with $\beta>0$, then the fact that $f(s) \geq 0$ for all $s \in \mathbf{R}$ yields $u>0$ in $\mathbf{R}$. Since $u(x) \rightarrow 0$ as $|x| \rightarrow \infty$, let $x_{0} \in \mathbf{R}$ be a maximum point of $u$. As in the proof of Lemma 2.2, from $\beta \in[0,1]$ and $f(s)>0$ for $s>0$ due to (f4), we get

$$
0<V_{0} \leq V\left(x_{0}\right) \leq \frac{\beta f\left(u\left(x_{0}\right)\right)}{u\left(x_{0}\right)} \leq \frac{f\left(u\left(x_{0}\right)\right)}{u\left(x_{0}\right)} .
$$

By (f2), we may find a $\delta_{1}>0$, which is independent of $\beta$ and $u$, so that

$$
\delta_{1} \leq u\left(x_{0}\right)=\|u\|_{L^{\infty}} \leq\|u\|_{\eta_{1}}
$$

for all $u \in X_{\eta_{1}} \backslash\{0\}$ and $\beta \in(0,1]$ with $u=\beta \mathcal{L}^{ \pm}(u)$. Therefore, selecting an $r_{0} \in\left(0, \delta_{1}\right)$, we see that

$$
\left(\mathrm{id}-\beta \mathcal{L}^{ \pm}\right)(u) \neq 0
$$

for all $u \in \partial B_{r_{0}}(0)$ and for all $\beta \in(0,1]$. Thus the lemma holds.

To show ii), we need some preparations. From (V3), we may select a $\kappa_{0}>0$ so that

$$
\left[-3 \kappa_{0}, 3 \kappa_{0}\right] \subset\left[V_{\infty}-V>0\right]:=\left\{x \in \mathbf{R} \mid V_{\infty}-V(x)>0\right\} .
$$

Next choose a $\varphi_{0} \in C_{0}^{\infty}(\mathbf{R})$ satisfying

$$
\begin{aligned}
& \varphi_{0}(-x)=\varphi_{0}(x), \quad 0 \leq \varphi_{0} \leq 1 \quad \text { in } \mathbf{R}, \quad \varphi_{0}^{\prime}(x) \leq 0 \quad \text { in }[0, \infty), \\
& \varphi_{0}(x)=1 \quad \text { if } 0 \leq x \leq \kappa_{0}, \quad \varphi_{0}(x)=0 \quad \text { if } 2 \kappa_{0} \leq x .
\end{aligned}
$$

Then we first prove

Lemma 2.6. There exists a $\tilde{t}=\tilde{t}\left(f, V_{\infty}\right)>0$ such that

$$
\frac{\kappa_{0}^{2}}{4 \Lambda} t \leq\|u\|_{L^{\infty}\left(\left[-\kappa_{0}, \kappa_{0}\right]\right)} \leq\|u\|_{X_{\eta_{1}}} \quad \text { for each } t \geq \tilde{t} \quad \text { and } \quad u \in \mathcal{S}_{t}^{ \pm}
$$

where

$$
\mathcal{S}_{t}^{ \pm}:=\left\{u \in X_{\eta_{1}} \mid-\mathcal{M}_{\lambda, \Lambda}^{ \pm}\left(u^{\prime \prime}\right)+V(x) u=f(u)+t \varphi_{0}\right\} .
$$

Proof. By (f2) and (f3), there exists a $c\left(f, V_{\infty}\right)>0$ such that

$$
\inf _{0 \leq s}\left(\frac{f(s)}{s}-V_{\infty}\right) s \geq-c\left(f, V_{\infty}\right)
$$

Choose a $\tilde{t}=\tilde{t}\left(f, V_{\infty}\right)>0$ so that if $t \geq \tilde{t}$, then $-c\left(f, V_{\infty}\right)+t \geq t / 2$. For this $\tilde{t}$, we shall prove that (2.11) holds.

Let $t \geq \tilde{t}$ and $u \in \mathcal{S}_{t}^{ \pm}$. Since $t>0$, we have $u \not \equiv 0$. Thus $u>0$ in $\mathbf{R}$ due to $f(s) \geq 0$ in $\mathbf{R}$ and the strong maximum principle. Hence, (V3) yields

$$
\begin{aligned}
-\mathcal{M}_{\lambda, \Lambda}^{ \pm}\left(u^{\prime \prime}\right) & =f(u)+t \varphi_{0}-V(x) u=\left(\frac{f(u)}{u}-V(x)\right) u+t \varphi_{0} \\
& \geq\left(\frac{f(u)}{u}-V_{\infty}\right) u+t \varphi_{0} \geq-c\left(f, V_{\infty}\right)+t \varphi_{0} \quad \text { in } \mathbf{R} .
\end{aligned}
$$


By the definition of $\varphi_{0}$, we see

$$
-\mathcal{M}_{\lambda, \Lambda}^{ \pm}\left(u^{\prime \prime}\right) \geq \frac{t}{2} \quad \text { in }\left[-\kappa_{0}, \kappa_{0}\right]
$$

which implies

$$
u^{\prime \prime} \leq-\frac{t}{2 \Lambda} \quad \text { in }\left[-\kappa_{0}, \kappa_{0}\right]
$$

Integrating the inequality over $[x, y] \subset\left[-\kappa_{0}, \kappa_{0}\right]$, one has

$$
u^{\prime}(y) \leq u^{\prime}(x)-\frac{t}{2 \Lambda}(y-x) \text { for }-\kappa_{0} \leq x \leq y \leq \kappa_{0} .
$$

Now we divide our arguments into two cases:

Case 1 There exists an $x_{0} \in\left[-\kappa_{0}, 0\right]$ such that $u^{\prime}\left(x_{0}\right) \leq 0$.

Case $2 u^{\prime}>0$ in $\left[-\kappa_{0}, 0\right]$.

In Case 1 , we put $x=x_{0}$ and integrate (2.12) in $y$ over $\left[x_{0}, \kappa_{0}\right]$ to obtain

$$
u\left(\kappa_{0}\right) \leq u\left(x_{0}\right)+u^{\prime}\left(x_{0}\right)\left(\kappa_{0}-x_{0}\right)-\frac{t}{4 \Lambda}\left(\kappa_{0}-x_{0}\right)^{2} \leq u\left(x_{0}\right)-\frac{t}{4 \Lambda}\left(\kappa_{0}-x_{0}\right)^{2} .
$$

Hence,

$$
\|u\|_{L^{\infty}\left(\left[-\kappa_{0}, \kappa_{0}\right]\right)} \geq u\left(x_{0}\right) \geq u\left(\kappa_{0}\right)+\frac{t}{4 \Lambda}\left(\kappa_{0}-x_{0}\right)^{2} \geq \frac{t}{4 \Lambda}\left(\kappa_{0}-x_{0}\right)^{2} \geq \frac{\kappa_{0}^{2}}{4 \Lambda} t .
$$

Thus (2.11) holds.

In Case 2, putting $y=0$ in (2.12), it follows that

$$
-\frac{t}{2 \Lambda} x \leq u^{\prime}(x) \text { for every } x \in\left[-\kappa_{0}, 0\right] \text {. }
$$

Integrating this inequality over $\left[-\kappa_{0}, 0\right]$, we obtain

$$
\frac{\kappa_{0}^{2}}{4 \Lambda} t \leq u(0)-u\left(-\kappa_{0}\right)<u(0) \leq\|u\|_{L^{\infty}\left(\left[-\kappa_{0}, \kappa_{0}\right]\right)} .
$$

Thus (2.11) holds and we complete the proof.

Next, we shall prove some properties of elements in $\mathcal{S}_{t}^{ \pm}$.

Lemma 2.7. Let $t \geq 0$ and $u \in \mathcal{S}_{t}^{ \pm} \backslash\{0\}$. Then either

(i) There exists an $x_{0} \in \mathbf{R}$ such that $u^{\prime}(y)<0<u^{\prime}(x)$ for all $x<x_{0}<y$ or else

(ii) There are $y_{0}<0<z_{0}$ such that $u^{\prime}\left(y_{0}\right)=0=u^{\prime}\left(z_{0}\right)$ and $u^{\prime}(x) \neq 0$ if $x \neq y_{0}, z_{0}$.

In particular, every $u \in \mathcal{S}_{t}^{ \pm} \backslash\{0\}$ has only one maximum point in $\mathbf{R}$.

Proof. For $u \in \mathcal{S}_{t}^{ \pm} \backslash\{0\}$, it suffices to prove the following claim:

Claim: If $u^{\prime}\left(z_{0}\right)=0$ holds for some $z_{0} \geq 0$, then $u^{\prime}(x)<0$ for every $x>z_{0}$. Similarly, if $u^{\prime}\left(y_{0}\right)=0$ holds for $y_{0} \leq 0$, then $u^{\prime}(x)>0$ for all $x<y_{0}$. In particular, each $u \in \mathcal{S}_{t}^{ \pm} \backslash\{0\}$ has at most one critical point in $[0, \infty]$ (resp. $(-\infty, 0])$. 
We first remark that since $u(-x)$ satisfies the same type of equation by (2.10) and (V1)-(V3), it is enough to prove the first assertion. To this end, suppose that $z_{0} \geq 0$ satisfies $u^{\prime}\left(z_{0}\right)=0$ and set, for all $x \in \mathbf{R}$,

$$
\tilde{u}(x)=u\left(z_{0}+|x|\right), \quad \tilde{V}(x)=V\left(z_{0}+|x|\right) \quad \text { and } \quad \tilde{\varphi}_{0}(x)=\varphi_{0}\left(z_{0}+|x|\right) .
$$

Then, since $u^{\prime}\left(z_{0}\right)=0$ and $z_{0} \geq 0, \tilde{u} \in C^{1}(\mathbf{R}) \cap C^{2}(\mathbf{R} \backslash\{0\}), \tilde{u}(x) \rightarrow 0$ as $|x| \rightarrow \infty$. Moreover $\tilde{u}, \tilde{V}$ and $\tilde{\varphi}_{0}$ are even and $\tilde{V}^{\prime}(x) \geq 0, \tilde{\varphi}_{0}^{\prime}(x) \leq 0$ for a.a. $x \geq 0$ and

$$
-\mathcal{M}_{\lambda, \Lambda}^{ \pm}\left(\tilde{u}^{\prime \prime}\right)+\tilde{V} \tilde{u}=f(\tilde{u})+t \tilde{\varphi}_{0} \quad \text { in } \quad \mathbf{R} \backslash\{0\} .
$$

Furthermore, by the differential equations and $u \in C^{2}(\mathbf{R})$, we have $\tilde{u} \in$ $C^{2}(\mathbf{R})$ and the equation above is satisfied in $\mathbf{R}$.

We shall prove Claim by the moving plane method. For $\lambda>0$, define $x_{\lambda}=2 \lambda-x, \Sigma_{\lambda}=\{x \in(0, \infty) \mid \lambda<x\}$ and

$$
u_{\lambda}(x)=\tilde{u}\left(x_{\lambda}\right)-\tilde{u}(x), \quad \varphi_{\lambda}(x)=\tilde{\varphi}_{0}\left(x_{\lambda}\right)-\tilde{\varphi}_{0}(x) .
$$

Since

$$
\begin{aligned}
-\mathcal{M}_{\lambda, \Lambda}^{ \pm}\left(\tilde{u}^{\prime \prime}\right)\left(x_{\lambda}\right)+\tilde{V}\left(x_{\lambda}\right) \tilde{u}\left(x_{\lambda}\right) & =f\left(\tilde{u}\left(x_{\lambda}\right)\right)+t \tilde{\varphi}_{0}\left(x_{\lambda}\right) \\
-\mathcal{M}_{\lambda, \Lambda}^{ \pm}\left(\tilde{u}^{\prime \prime}\right)(x)+\tilde{V}(x) \tilde{u}(x) & =f(\tilde{u}(x))+t \tilde{\varphi}_{0}(x),
\end{aligned}
$$

we have

$$
\begin{aligned}
& -\left(\mathcal{M}_{\lambda, \Lambda}^{ \pm}\left(\tilde{u}^{\prime \prime}\right)\left(x_{\lambda}\right)-\mathcal{M}_{\lambda, \Lambda}^{ \pm}\left(\tilde{u}^{\prime \prime}\right)(x)\right)+\left(\tilde{V}\left(x_{\lambda}\right)-\tilde{V}(x)\right) \tilde{u}\left(x_{\lambda}\right)+\tilde{V}(x) u_{\lambda} \\
= & f\left(\tilde{u}\left(x_{\lambda}\right)\right)-f(\tilde{u}(x))+t\left(\tilde{\varphi}_{0}\left(x_{\lambda}\right)-\tilde{\varphi}_{0}(x)\right) .
\end{aligned}
$$

Noting (2.8), $\left|x_{\lambda}\right| \leq|x|, \tilde{V}\left(x_{\lambda}\right) \leq \tilde{V}(x)$ and $\tilde{\varphi}_{0}(x) \leq \tilde{\varphi}_{0}\left(x_{\lambda}\right)$ for all $x \in \Sigma_{\lambda}$, we have

$$
-\mathcal{M}_{\lambda, \Lambda}^{-}\left(u_{\lambda}^{\prime \prime}\right)+\tilde{V}(x) u_{\lambda} \geq f\left(\tilde{u}\left(x_{\lambda}\right)\right)-f(\tilde{u}(x)) \text { in } \quad \Sigma_{\lambda}
$$

Moreover, from

$$
\begin{aligned}
f\left(\tilde{u}\left(x_{\lambda}\right)\right)-f(\tilde{u}(x)) & =\int_{0}^{1} f^{\prime}\left(\tilde{u}(x)+\theta u_{\lambda}(x)\right) \mathrm{d} \theta u_{\lambda}(x) \\
& =: \quad g_{\lambda}(x) u_{\lambda}(x),
\end{aligned}
$$

we have

$$
-\mathcal{M}_{\lambda, \Lambda}^{-}\left(u_{\lambda}^{\prime \prime}\right)+\left(\tilde{V}(x)-g_{\lambda}(x)\right) u_{\lambda}(x) \geq 0 \quad \text { in } \quad \Sigma_{\lambda} .
$$

Since $\tilde{u}>0$ in $\mathbf{R}, f^{\prime}(0)=0$ by (f2) and $\tilde{u}(x) \rightarrow 0$ as $x \rightarrow \infty$, it is not difficult to see that the strong maximum principle implies that for all $\lambda$ sufficiently large,

$$
u_{\lambda}>0 \quad \text { in } \quad \Sigma_{\lambda}, \quad u_{\lambda}^{\prime}(\lambda)=-2 \tilde{u}^{\prime}(\lambda)=-2 u^{\prime}\left(z_{0}+\lambda\right)>0 .
$$

Next, set

$$
\lambda_{*}=\inf \left\{\lambda>0 \mid u_{\tilde{\lambda}}>0 \text { in } \Sigma_{\tilde{\lambda}} \text { for all } \tilde{\lambda}>\lambda\right\} .
$$


From the above observation, we have $0 \leq \lambda_{*}<\infty$. In addition, notice that if $u_{\lambda} \geq 0$ in $\Sigma_{\lambda}$, then

$$
\begin{aligned}
0 & \leq-\mathcal{M}_{\lambda, \Lambda}^{-}\left(u_{\lambda}^{\prime \prime}\right)+\left(\tilde{V}(x)-g_{\lambda}(x)\right) u_{\lambda}(x) \\
& \leq-\mathcal{M}_{\lambda, \Lambda}^{-}\left(u_{\lambda}^{\prime \prime}\right)+\left(\tilde{V}(x)-g_{\lambda}(x)\right)_{+} u_{\lambda}(x) \text { in } \Sigma_{\lambda} .
\end{aligned}
$$

In particular, since $u_{\lambda_{*}} \geq 0$ in $\Sigma_{\lambda_{*}}$, the strong maximum principle yields either

(i) $u_{\lambda_{*}}>0$ in $\Sigma_{\lambda_{*}}, u_{\lambda_{*}}^{\prime}\left(\lambda_{*}\right)>0$

or else

(ii) $u_{\lambda_{*}} \equiv 0$ in $\Sigma_{\lambda_{*}}$.

Next we prove that if $\mu>0$ and $u_{\mu}>0$ in $\Sigma_{\mu}$ hold, then there exists an $\epsilon_{\mu}>0$ such that $u_{\tilde{\mu}}>0$ in $\Sigma_{\tilde{\mu}}$ provided $|\mu-\tilde{\mu}|<\epsilon_{\mu}$. To see this, we remark that

$$
u_{\tilde{\mu}} \rightarrow u_{\mu} \quad \text { in } C_{\mathrm{loc}}^{1}([\mu, \infty)) \text {. }
$$

Since $u_{\mu}^{\prime}(\mu)>0$ holds due to $u_{\mu}>0$ in $\Sigma_{\mu}$ and the strong maximum principle, for sufficiently small $\epsilon_{\mu}$, we observe that $|\mu-\tilde{\mu}|<\epsilon_{\mu}$ implies $u_{\tilde{\mu}}>0$ in $\left(\tilde{\mu}, R_{\epsilon}\right)$ where $R_{\epsilon}>0$ is chosen so that $x \geq R_{\epsilon}$ implies $g_{\tilde{\mu}}(x) \leq V_{0} / 2$.

From

$$
-\mathcal{M}_{\lambda, \Lambda}^{-}\left(u_{\tilde{\mu}}^{\prime \prime}\right)+\left(V-V_{0} / 2\right) u_{\tilde{\mu}} \geq 0 \quad \text { in } \quad\left[R_{\epsilon}, \infty\right), \quad u_{\tilde{\mu}}(x) \rightarrow 0 \quad \text { as } x \rightarrow \infty
$$

and the strong maximum principle, $u_{\tilde{\mu}}$ cannot take a non-positive minimum. Hence $|\mu-\tilde{\mu}|<\epsilon_{\mu}$ implies $u_{\tilde{\mu}}>0$ in $\Sigma_{\tilde{\mu}}$ and $u_{\tilde{\mu}}^{\prime}(\tilde{\mu})>0$.

By this claim we see that if $u_{\lambda_{*}}>0$ in $\Sigma_{\lambda_{*}}$, then $\lambda_{*}=0$. Thus, $\lambda_{*}=0$ holds provided (i) occurs. Moreover, we also see from $(2.13)$ that $\tilde{u}^{\prime}(x)<0$ for all $x>0$.

On the other hand, let us consider the case $\lambda_{*}>0$ and $u_{\lambda_{*}} \equiv 0$ in $\Sigma_{\lambda_{*}}$. In this case, we notice that $-2 \tilde{u}^{\prime}(\lambda)=u_{\lambda}^{\prime}(\lambda)>0$ for all $\lambda>\lambda_{*}$ and $\tilde{u}\left(2 \lambda_{*}-x\right)=\tilde{u}(x)$ for all $x \geq \lambda_{*}$. Since $\tilde{u}^{\prime}(0)=0$, we have $\tilde{u}^{\prime}\left(2 \lambda_{*}\right)=0$, which is a contradiction. Hence, (ii) only occurs when $\lambda_{*}=0$ and it follows from (2.13) that $\tilde{u}^{\prime}(x)<0$ for all $x>0$.

By the above observations, we obtain $\lambda_{*}=0$ and $\tilde{u}^{\prime}(x)<0$ for all $x>0$, which implies $u^{\prime}(x)<0$ for all $x>z_{0}$. Thus we complete the proof.

Lemma 2.8. There exists an $M_{0}>0$ such that

$$
\|u\|_{L^{\infty}(\mathbf{R})} \leq M_{0} \quad \text { for each } u \in \mathcal{S}_{t}^{ \pm} \quad \text { and } \quad t \geq 0 .
$$

Proof. We argue by contradiction and suppose that there are $\left(s_{n}\right) \subset[0, \infty)$ and $u_{n} \in \mathcal{S}_{s_{n}}^{ \pm}$such that $\tau_{n}:=\left\|u_{n}\right\|_{L^{\infty}(\mathbf{R})} \rightarrow \infty$. Thanks to Lemma 2.7, let $\left(x_{n}\right) \subset \mathbf{R}$ be a unique maximum point of $\left(u_{n}\right)$ and set

$$
\begin{aligned}
v_{n}(x) & :=\frac{1}{\tau_{n}} u_{n}\left(x_{n}+\sqrt{\frac{\tau_{n}}{f\left(\tau_{n}\right)}} x\right), \quad \varphi_{n}(x):=\varphi_{0}\left(x_{n}+\sqrt{\frac{\tau_{n}}{f\left(\tau_{n}\right)}} x\right), \\
V_{n}(x) & :=V\left(x_{n}+\sqrt{\frac{\tau_{n}}{f\left(\tau_{n}\right)}} x\right) .
\end{aligned}
$$


Then $v_{n}$ satisfies

$v_{n}(x) \leq v_{n}(0)=1, \quad-\mathcal{M}_{\lambda, \Lambda}^{ \pm}\left(v_{n}^{\prime \prime}\right)+\frac{\tau_{n}}{f\left(\tau_{n}\right)} V_{n} v_{n}=\frac{f\left(\tau_{n} v_{n}\right)}{f\left(\tau_{n}\right)}+\frac{s_{n}}{f\left(\tau_{n}\right)} \varphi_{n} \quad$ in $\mathbf{R}$.

Recalling Lemma 2.6, we have

$$
\frac{\kappa_{0}^{2}}{4 \Lambda} s_{n}-\frac{\kappa_{0}^{2}}{4 \Lambda} \tilde{t} \leq\left\|u_{n}\right\|_{L^{\infty}(\mathbf{R})}=\tau_{n} \quad \text { for all } n .
$$

Hence, by (f3), $s_{n} / f\left(\tau_{n}\right) \rightarrow 0$ as $n \rightarrow \infty$. Moreover, noting that $f(s)$ is increasing in $[0, \infty)$ by (f4), it follows from $v_{n}(x) \leq 1$ that

$$
\left|\frac{f\left(\tau_{n} v_{n}\right)}{f\left(\tau_{n}\right)}\right| \leq 1 \quad \text { in } \mathbf{R} \text {. }
$$

Noting $v_{n}^{\prime}(0)=0$ and $\tau_{n} / f\left(\tau_{n}\right) \rightarrow 0$, we may extract a subsequence (still denoted by $(n))$ such that

$$
v_{n} \rightarrow v_{0} \quad \in C_{\mathrm{loc}}^{1}(\mathbf{R}), \quad 0 \leq v_{0} \leq 1 \quad \text { in } \mathbf{R}, \quad v_{0}(0)=1, \quad v_{0}^{\prime}(0)=0 .
$$

Furthermore, by $0 \leq v_{n} \leq 1$ and (f3), we have

$$
\frac{f\left(\tau_{n} v_{n}\right)}{f\left(\tau_{n}\right)} \rightarrow \bar{f}\left(v_{0}\right) \text { in } C_{\mathrm{loc}}\left(\left[v_{0}>0\right]\right) .
$$

Since $0 \in\left[v_{0}>0\right]$, let $I$ be a component of $\left[v_{0}>0\right]$ satisfying $0 \in I$. Then we have

$$
v_{n} \rightarrow v_{0} \quad \text { in } C_{\text {loc }}^{2}(I), \quad-\mathcal{M}_{\lambda, \Lambda}^{ \pm}\left(v_{0}^{\prime \prime}\right)=\bar{f}\left(v_{0}\right) \quad \text { in } I .
$$

When $I=\left(-c_{2}, c_{1}\right)$ and $c_{1}<\infty$, since $v_{0}^{\prime}(0)=0, v_{0}(0)=1, \bar{f} \geq 0$, $\bar{f}(1)=1>0$ and $v_{0}\left(c_{1}\right)=0$, we observe that $v_{0}^{\prime}\left(c_{1}\right)<0$, however, this contradicts $0 \leq v_{0} \leq 1$ in $\mathbf{R}$.

On the other hand, if $I=\left(-c_{2}, \infty\right)$, then by $v_{0}^{\prime}(0)=0, v_{0}(0)=1$ and $-\mathcal{M}_{\lambda, \Lambda}^{ \pm}\left(v_{0}^{\prime \prime}\right)=\bar{f}\left(v_{0}\right)$, we observe that $v_{0}$ must hit a zero at some $x_{0}>0$ with $v_{0}^{\prime}\left(x_{0}\right)<0$, however this contradicts $0 \leq v_{0} \leq 1$ again. Hence, Lemma 2.8 holds and we complete the proof.

The next proposition is a key in order to prove $\operatorname{deg}_{X_{\eta_{1}}}\left(\mathrm{id}-\mathcal{L}^{ \pm}, B_{r_{1}}(0), 0\right)=$ 0 for some $r_{1}>r_{0}$.

Proposition 2.9. There exists an $M_{1}>0$ such that

$$
\|u\|_{X_{\eta_{1}}} \leq M_{1} \quad \text { for each } u \in \mathcal{S}_{t}^{ \pm} \quad \text { and } \quad t \geq 0 .
$$

Assuming Proposition 2.9, we first prove Theorem 1.1. Before the proof, we remark that for every $t \geq 0$ and $v \in X_{\eta_{1}}$, the equations

$$
-\mathcal{M}_{\lambda, \Lambda}^{ \pm}\left(u^{\prime \prime}\right)+V(x) u=f(v)+t \varphi_{0} \quad \text { in } \mathbf{R}
$$

have unique solutions in $X_{\eta_{1}}$. Indeed, we may prove this claim in a similar way to the proof of Lemma 2.2 thanks to $\varphi_{0} \in C_{0}^{\infty}(\mathbf{R})$. Thus, we denote by $F^{ \pm}(t, v)$ these unique solutions. Furthermore, we may show that the maps $(t, v) \mapsto F^{ \pm}(t, v):[0, \infty) \times X_{\eta_{1}} \rightarrow X_{\eta_{1}}$ are compact as in Lemma 2.4.

Now we prove Theorem 1.1. 
Proof of Theorem 1.1. Choose $r_{1}:=M_{1}+\frac{\kappa_{0}^{2}}{4 \Lambda} \tilde{t}+r_{0}$ where $M_{1}, \tilde{t}$ and $r_{0}$ appear in Proposition 2.9 and Lemmas 2.6 and 2.5. We first claim that

$$
u-F^{ \pm}\left(t_{1}, u\right) \neq 0 \text { in } \overline{B_{r_{1}}}
$$

where $t_{1}>\tilde{t}$ is chosen so that $\frac{\kappa_{0}^{2}}{4 \Lambda} t_{1}>r_{1}$. Indeed, let $u \in \overline{B_{r_{1}}}$ satisfy $u-$ $F^{ \pm}\left(t_{1}, u\right)=0$. Noting $u \in \mathcal{S}_{t_{1}}^{ \pm}$and $t_{1}>\tilde{t}$, Lemma 2.6 gives a contradiction:

$$
r_{1} \geq\|u\|_{X_{\eta_{1}}} \geq\|u\|_{L^{\infty}(\mathbf{R})} \geq \frac{\kappa_{0}^{2}}{4 \Lambda} t_{1}>r_{1} .
$$

Hence, (2.14) holds.

Since Proposition 2.9 and the choice of $r_{1}$ imply

$$
u-F^{ \pm}(t, u) \neq 0 \quad \text { on } \partial B_{r_{1}} \quad \text { for every } t \geq 0,
$$

it is easily seen from (2.14) and the homotopy invariance of degree that

$$
\operatorname{deg}_{X_{\eta_{1}}}\left(\mathrm{id}-\mathcal{L}^{ \pm}, B_{r_{1}}(0), 0\right)=0 .
$$

Combining this with Lemma 2.5, we obtain

$$
\operatorname{deg}_{X_{\eta_{1}}}\left(\mathrm{id}-\mathcal{L}^{ \pm}, A_{r_{0}, r_{1}}, 0\right)=-1
$$

and solutions of (1.1) in $A_{r_{0}, r_{1}}$. This completes the proof.

Before proceeding to the proof of Proposition 2.9, we remark the following fact on the function $g_{\infty}(s):=f(s)-V_{\infty} s$, which will be used below.

Fact: There exists a unique $s_{\infty}>0$ such that

$$
g_{\infty}(s)<0=g_{\infty}\left(s_{\infty}\right)<g_{\infty}(t) \text { for all } 0<s<s_{\infty}<t .
$$

This fact follows from (f1)-(f4). In fact, for sufficiently small $s>0$, by (f2), we get $g_{\infty}(s)<0$. On the other hand, (f3) yields $g_{\infty}(s) \rightarrow \infty$ as $s \rightarrow \infty$, hence, there exists an $s_{\infty}>0$ so that $g_{\infty}\left(s_{\infty}\right)=0$. Moreover, from

$$
g_{\infty}(s)=s\left(\frac{f(s)}{s}-V_{\infty}\right)
$$

and (f4), we see that (2.15) holds.

Now we prove Proposition 2.9.

Proof of Proposition 2.9. We argue indirectly and suppose that there exists $\left(s_{n}, u_{n}\right) \in[0, \infty) \times X_{\eta_{1}}$ such that $u_{n} \in \mathcal{S}_{s_{n}}^{ \pm}$and $\left\|u_{n}\right\|_{X_{\eta_{1}}} \rightarrow \infty$. Remark that $u_{n}$ satisfies

$$
-\mathcal{M}_{\lambda, \Lambda}^{ \pm}\left(u_{n}^{\prime \prime}\right)+V u_{n}=f\left(u_{n}\right)+s_{n} \varphi_{0} \quad \text { in } \mathbf{R} .
$$

By Lemma 2.7, $u_{n}$ has only one maximum point and denote it by $x_{n}$. Our first aim is to show

$$
\left(x_{n}\right) \text { is bounded. }
$$


To prove (2.16), suppose that $x_{n} \rightarrow \infty$. We may assume $3 \kappa_{0}<x_{n}$. Setting

$$
v_{n}(x):=u_{n}\left(x+x_{n}\right), \quad V_{n}(x):=V\left(x+x_{n}\right), \quad \varphi_{n}(x):=\varphi_{0}\left(x+x_{n}\right),
$$

we see $\varphi_{n} \equiv 0$ in $[0, \infty)$ thanks to $3 \kappa_{0}<x_{n}$. Furthermore, by Lemma 2.7, we have

$$
\begin{aligned}
& -\mathcal{M}_{\lambda, \Lambda}^{ \pm}\left(v_{n}^{\prime \prime}\right)+V_{n} v_{n}=f\left(v_{n}\right)+s_{n} \varphi_{n} \quad \text { in } \mathbf{R}, \quad v_{n}(0)=\max _{\mathbf{R}} v_{n}>0, \\
& v_{n}^{\prime}(y) \leq 0 \leq v_{n}^{\prime}(x) \quad \text { for } x<0<y, \quad V_{n} \rightarrow V_{\infty}, \quad s_{n} \varphi_{n} \rightarrow 0 \quad \text { in } C_{\mathrm{loc}}(\mathbf{R}) .
\end{aligned}
$$

In the sequel, we divide our arguments into several steps.

Step 1: One has

$$
v_{n} \rightarrow \omega_{ \pm} \quad \text { strongly in } C_{\text {loc }}^{2}(\mathbf{R})
$$

where $\omega_{ \pm}$are unique solutions of (2.1) and (2.2) (see Proposition 2.1).

We first notice that $\left(v_{n}\right)$ is bounded in $L^{\infty}(\mathbf{R})$ due to Lemma 2.8. Combining with $V_{n} \rightarrow V_{\infty}$ and $s_{n} \varphi_{n} \rightarrow 0$ in $C_{\text {loc }}(\mathbf{R})$, we may extract a subsequence (still denoted by $(n))$ so that

$$
\begin{aligned}
& v_{n} \rightarrow v_{0} \quad \text { in } C_{\mathrm{loc}}^{2}(\mathbf{R}), \quad-\mathcal{M}_{\lambda, \Lambda}^{ \pm}\left(v_{0}^{\prime \prime}\right)+V_{\infty} v_{0}=f\left(v_{0}\right) \quad \text { in } \mathbf{R}, \\
& v_{0}(0)=\max _{\mathbf{R}} v_{0}, \quad 0 \leq v_{0} \quad \text { in } \mathbf{R}, \quad v_{0}^{\prime}(y) \leq 0 \leq v_{0}^{\prime}(x) \quad \text { for } x<0<y .
\end{aligned}
$$

By $v_{n}(0)=\max _{\mathbf{R}} v_{n}$, we have $v_{n}^{\prime \prime}(0) \leq 0$. Since $v_{n}(0)>0$ and $\varphi_{n} \equiv 0$ on $[0, \infty)$, we get

$$
f\left(v_{n}(0)\right)=-\mathcal{M}_{\lambda, \Lambda}^{ \pm}\left(v_{n}^{\prime \prime}(0)\right)+V_{n}(0) v_{n}(0) \geq V_{n}(0) v_{n}(0),
$$

which implies

$$
V_{n}(0) \leq \frac{f\left(v_{n}(0)\right)}{v_{n}(0)}
$$

By $V_{n}(0) \rightarrow V_{\infty}$ and (f2), we may find a $\delta_{0}>0$ so that $v_{n}(0) \geq \delta_{0}$ for all $n$. Thus $v_{0}(0) \geq \delta_{0}$ and $v_{0}>0$ in $\mathbf{R}$. Now from $v_{0}^{\prime}(y) \leq 0$ in $[0, \infty)$, one has

$$
v_{0, \infty}:=\lim _{x \rightarrow \infty} v_{0}(y) \geq 0 .
$$

Since $-\mathcal{M}_{\lambda, \Lambda}^{ \pm}\left(v_{0}^{\prime \prime}\right)=g_{\infty}\left(v_{0}\right)$ in $\mathbf{R}$, it follows from (2.15) that

$$
\text { either } v_{0, \infty}=0 \text { or } v_{0, \infty}=s_{\infty}>0 \text {. }
$$

If $v_{0, \infty}=0$, then by Proposition 2.1, we have $v_{0}=\omega_{ \pm}$and Step 1 holds.

Now we assume $v_{0, \infty}=s_{\infty}$. By $v_{0}^{\prime} \leq 0$ in $[0, \infty)$ and (2.15), we have $v_{0} \geq s_{\infty}$ in $[0, \infty)$ and

$$
-\mathcal{M}_{\lambda, \Lambda}^{ \pm}\left(v_{0}^{\prime \prime}\right)=g_{\infty}\left(v_{0}\right) \geq 0 \quad \text { on }[0, \infty) .
$$

Moreover, if $v_{0}(0)>s_{\infty}$, then the strict inequality holds at $x=0$. However, this contradicts facts $v_{0}^{\prime}(0)=0>v_{0}^{\prime \prime}(0), v_{0}^{\prime \prime}(x) \leq 0$ for $x \in[0, \infty)$ and $v_{0}(x) \rightarrow s_{\infty}$ as $x \rightarrow \infty$. Thus we get $v_{0} \equiv s_{\infty}$ in $[0, \infty)$. 
Next, we put

$$
\begin{aligned}
& E_{n,+}(x):=\frac{\Lambda}{2}\left(v_{n}^{\prime}(x)\right)^{2}+F\left(v_{n}(x)\right)-\frac{V_{n}}{2} v_{n}^{2} \text { for } \mathcal{M}_{\lambda, \Lambda}^{+}, \\
& E_{n,-}(x):=\frac{\lambda}{2}\left(v_{n}^{\prime}(x)\right)^{2}+F\left(v_{n}(x)\right)-\frac{V_{n}}{2} v_{n}^{2} \quad \text { for } \mathcal{M}_{\lambda, \Lambda}^{-} .
\end{aligned}
$$

We also put $h_{n}(x):=V_{n}(x)-f\left(v_{n}(x)\right) / v_{n}(x)$. Recalling $V_{n}(x)=V\left(x+x_{n}\right)$ and $x_{n} \rightarrow \infty$, we may assume that $V_{n}^{\prime}(x) \geq 0$ in $(0, \infty)$. Notice also that $v_{n}$ is strictly decreasing in $(0, \infty)$ by Lemma 2.7. Hence (f4) yields that $h_{n}(x)$ is strictly increasing in $[0, \infty)$. Since $v_{n}^{\prime \prime}(0) \leq 0$ and $\mathcal{M}_{\lambda, \Lambda}^{ \pm}\left(v_{n}^{\prime \prime}\right)=v_{n} h_{n}$ in $[0, \infty)$, we see $h_{n}(0) \leq 0$. Noting $h_{n}(x) \rightarrow V_{\infty}>0$ as $x \rightarrow \infty$, there exists a unique $z_{n}^{ \pm} \geq 0$ such that $h_{n}\left(z_{n}^{ \pm}\right)=0$. Therefore, one has

$$
v_{n}^{\prime \prime}(x)<0<v_{n}^{\prime \prime}(y) \text { for } 0 \leq x<z_{n}^{ \pm}<y .
$$

Moreover, taking a subsequence if necessary, we may assume $v_{n}\left(z_{n}^{ \pm}\right) \rightarrow \tilde{s} \geq 0$ since $v_{n}\left(z_{n}^{ \pm}\right)$is bounded. Noting $V_{n}\left(z_{n}^{ \pm}\right) \rightarrow V_{\infty}$ as $n \rightarrow \infty$ and letting $n \rightarrow \infty$ in $h_{n}\left(z_{n}^{ \pm}\right)=0$, it follows from (f2) that

$$
\tilde{s}>0 \quad \text { and } \quad V_{\infty}=\frac{f(\tilde{s})}{\tilde{s}} \text {. }
$$

Thus by (2.15), we obtain $\tilde{s}=s_{\infty}$ and $v_{n}\left(z_{n}^{ \pm}\right) \rightarrow s_{\infty}$. Recalling $v_{n}^{\prime \prime}(x) \geq 0$ for $x \geq z_{n}^{ \pm}, V_{n}^{\prime} \geq 0$ in $[0, \infty)$ and $\varphi_{n} \equiv 0$ in $[0, \infty)$, we have

$$
\begin{aligned}
& E_{n,+}^{\prime}(x)=v_{n}^{\prime}(x)\left(\Lambda v_{n}^{\prime \prime}+f\left(v_{n}\right)-V_{n} v_{n}\right)-\frac{V_{n}^{\prime}}{2} v_{n}^{2}=-\frac{V_{n}^{\prime}}{2} v_{n}^{2} \leq 0 \quad \text { in }\left[z_{n}^{+}, \infty\right), \\
& E_{n,-}^{\prime}(x)=-\frac{V_{n}^{\prime}}{2} v_{n}^{2} \leq 0 \quad \text { in }\left[z_{n}^{-}, \infty\right) .
\end{aligned}
$$

Thanks to $E_{n, \pm}(x) \rightarrow 0$ as $x \rightarrow \infty$, one sees $E_{n, \pm}\left(z_{n}^{ \pm}\right) \geq 0$. Since it follows from (2.15) that

$$
V_{n}\left(z_{n}^{ \pm}\right) \rightarrow V_{\infty}, \quad G_{\infty}\left(s_{\infty}\right)=\int_{0}^{s_{\infty}} g_{\infty}(s) \mathrm{d} s=\min _{[0, \infty)} G(s)<0
$$

we obtain

$$
\left(v_{n}^{\prime}\left(z_{n}^{ \pm}\right)\right)^{2} \geq \frac{2}{\Lambda}\left\{\frac{V_{n}\left(z_{n}^{ \pm}\right)}{2} v_{n}^{2}\left(z_{n}^{ \pm}\right)-F\left(v_{n}\left(z_{n}^{ \pm}\right)\right)\right\} \rightarrow-\frac{2}{\Lambda} G_{\infty}\left(s_{\infty}\right)>0
$$

By the fact that $\left(v_{n}^{\prime \prime}\right)$ is bounded in $[-1, \infty)$, we may find a $\delta_{1}, \delta_{2}>0$ so that

$$
\left|v_{n}^{\prime}(x)\right| \geq \delta_{1}>0 \quad \text { in }\left[z_{n}^{ \pm}-\delta_{2}, z_{n}^{ \pm}+\delta_{2}\right] .
$$

Due to this and the fact $v_{n}^{\prime}(0)=0$, shrinking $\delta_{2}>0$ if necessary, we may assume $z_{n}^{ \pm} \geq \delta_{2}>0$ for any $n$. Furthermore, by $v_{n}\left(z_{n}^{ \pm}\right) \rightarrow s_{\infty}$ and $v_{n}^{\prime} \leq 0$ in $[0, \infty)$, we obtain

$v_{n}(0) \geq v_{n}\left(z_{n}^{ \pm}-\delta_{2}\right)=v_{n}\left(z_{n}^{ \pm}\right)-\int_{z_{n}^{ \pm}-\delta_{2}}^{z_{n}^{ \pm}} v_{n}^{\prime}(x) \mathrm{d} x \geq v_{n}\left(z_{n}^{ \pm}\right)+\delta_{1} \delta_{2} \rightarrow s_{\infty}+\delta_{1} \delta_{2}$.

However, this contradicts $v_{0} \equiv s_{\infty}$ in $[0, \infty)$. Thus $v_{0, \infty}=0$ and Step 1 holds. 
To proceed further, we need some preparations. First, combining the monotonicity of $v_{n}$ with $(2.17)$, we can prove that

$$
v_{n} \rightarrow \omega_{ \pm} \text {strongly in } L^{\infty}(\mathbf{R}) .
$$

Moreover, by the differential equation, we also derive the uniform exponential decay at $x=\infty$ :

$$
v_{n}(x)+\left|v_{n}^{\prime}(x)\right| \leq c_{3} \exp \left(-c_{4} x\right) \quad \text { for all } x \geq 0 \quad \text { and } \quad n \geq 1
$$

where $c_{3}, c_{4}>0$ do not depend on $n$. Thus, using the same notation $z_{n}^{ \pm}$ to the above, namely, unique points satisfying $v_{n}^{\prime \prime}\left(z_{n}^{ \pm}\right)=0$ and $z_{n}^{ \pm} \geq 0$, we claim that $z_{n}^{ \pm} \rightarrow z^{ \pm}$where $z^{ \pm}$are unique points satisfying $z^{ \pm}>0$ and $\omega_{ \pm}^{\prime \prime}\left(z^{ \pm}\right)=0$. In fact, the unique existence of $z^{ \pm}$is ensured by Proposition 2.1. Furthermore, by (2.18), (V1), (f2), $\varphi_{n} \equiv 0$ in $[0, \infty)$ and $\omega_{ \pm}(x) \rightarrow 0$ as $|x| \rightarrow \infty$, there exist $n_{0}$ and $R_{0}>0$ such that if $n \geq n_{0}$ and $x \geq R_{0}$, then

$$
\mathcal{M}_{\lambda, \Lambda}^{ \pm}\left(v_{n}^{\prime \prime}\right)=V(x) v_{n}(x)-f\left(v_{n}(x)\right)>0,
$$

which yields $z_{n}^{ \pm} \leq R_{0}$. Moreover, by $\omega_{ \pm}^{\prime \prime}(0)<0$, we also observe that $z_{n}^{ \pm}$ never approaches to 0 . Thus, by the uniqueness of $z^{ \pm}$, we have $z_{n}^{ \pm} \rightarrow z^{ \pm}$ and we may assume $z_{n}^{ \pm}>0$.

Next, since $v_{n}$ is strictly increasing in $(-\infty, 0]$ and strictly decreasing in $[0, \infty)$, let $y_{n}^{ \pm}(s)$ and $z_{n}^{ \pm}(s)$ be inverse functions of $v_{n}$ satisfying $y_{n}^{ \pm}(s) \leq 0 \leq$ $z_{n}^{ \pm}(s)$ for $0<s \leq v_{n}(0)$. In particular, we have

$$
y_{n}^{ \pm}, z_{n}^{ \pm} \in C\left(\left(0, v_{n}(0)\right], \mathbf{R}\right), \quad v_{n}\left(y_{n}^{ \pm}(s)\right)=s=v_{n}\left(z_{n}^{ \pm}(s)\right) \quad \text { for } 0<s \leq v_{n}(0) .
$$

Moreover, $y_{n}^{ \pm}, z_{n}^{ \pm}$are smooth except for at most two points $s=v_{n}(0)$ and $s=v_{n}(y)$ where $v_{n}^{\prime}(y)=0$ and $y \neq 0$. Set $y_{n}^{ \pm}:=y_{n}^{ \pm}\left(v_{n}\left(z_{n}^{ \pm}\right)\right)$, namely, $y_{n}^{ \pm}<0$ and $v_{n}\left(y_{n}^{ \pm}\right)=v_{n}\left(z_{n}^{ \pm}\right)$hold. Moreover, by $\omega_{ \pm}(-x)=\omega_{ \pm}(x), y_{n}^{ \pm} \rightarrow-z^{ \pm}$as $n \rightarrow \infty$. Next, set

$$
\begin{aligned}
& E_{n, \infty,+}(x):=\frac{\Lambda}{2}\left(v_{n}^{\prime}(x)\right)^{2}+F\left(v_{n}(x)\right)-\frac{V_{\infty}}{2} v_{n}^{2}(x) \text { for } \mathcal{M}_{\lambda, \Lambda}^{+}, \\
& E_{n, \infty,-}(x):=\frac{\lambda}{2}\left(v_{n}^{\prime}(x)\right)^{2}+F\left(v_{n}(x)\right)-\frac{V_{\infty}}{2} v_{n}^{2}(x) \text { for } \mathcal{M}_{\lambda, \Lambda}^{-} .
\end{aligned}
$$

Remark that $E_{n, \infty, \pm}(x) \rightarrow 0$ as $|x| \rightarrow \infty$. Then we shall prove

Step 2: We have

$$
\begin{aligned}
& 0 \leq-E_{n, \infty,+}\left(z_{n}^{+}\right) \leq c \exp \left(-2 x_{n} \sqrt{\frac{V_{\infty}}{\Lambda}+\xi_{0}}\right) \\
& 0 \leq-E_{n, \infty,-}\left(z_{n}^{-}\right) \leq c \exp \left(-2 x_{n} \sqrt{\frac{V_{\infty}}{\lambda}+\xi_{0}}\right)
\end{aligned}
$$

where $c>0$ is independent of $n$ and $\xi_{0}>0$ the constant in (V3). 
First we notice that

$$
\begin{aligned}
& E_{n, \infty,+}^{\prime}(x)=v_{n}^{\prime}(x)\left(\Lambda v_{n}^{\prime \prime}+f\left(v_{n}\right)-V_{\infty} v_{n}\right) \\
& = \begin{cases}v_{n}^{\prime}\left[\left(V_{n}-V_{\infty}\right) v_{n}-s_{n} \varphi_{n}\right] & \text { if } v_{n}^{\prime \prime}(x) \geq 0, \\
v_{n}^{\prime}\left[\frac{\Lambda}{\lambda}\left(V_{n} v_{n}-f\left(v_{n}\right)-s_{n} \varphi_{n}\right)+f\left(v_{n}\right)-V_{\infty} v_{n}\right] & \text { if } v_{n}^{\prime \prime}(x)<0\end{cases}
\end{aligned}
$$

and

$$
\begin{aligned}
& E_{n, \infty,-}^{\prime}(x)=v_{n}^{\prime}(x)\left(\lambda v_{n}^{\prime \prime}+f\left(v_{n}\right)-V_{\infty} v_{n}\right) \\
& = \begin{cases}v_{n}^{\prime}\left[\left(V_{n}-V_{\infty}\right) v_{n}-s_{n} \varphi_{n}\right] & \text { if } v_{n}^{\prime \prime}(x) \geq 0, \\
v_{n}^{\prime}\left[\frac{\lambda}{\Lambda}\left(V_{n} v_{n}-f\left(v_{n}\right)-s_{n} \varphi_{n}\right)+f\left(v_{n}\right)-V_{\infty} v_{n}\right] & \text { if } v_{n}^{\prime \prime}(x)<0 .\end{cases}
\end{aligned}
$$

Since $v_{n}^{\prime \prime}>0$ in $\left(z_{n}^{ \pm}, \infty\right), v_{n}^{\prime} \leq 0$ in $[0, \infty)$ and $\varphi_{n} \equiv 0$ in $[0, \infty)$, we get

$$
\left(E_{n, \infty, \pm}\right)^{\prime}(x)=v_{n}^{\prime}\left(V_{n}-V_{\infty}\right) v_{n} \geq 0 \quad \text { in }\left(z_{n}^{ \pm}, \infty\right) .
$$

Hence, (V3), $z_{n}^{ \pm} \rightarrow z^{ \pm}>0$ and (2.19) give

$$
\begin{aligned}
0 & \leq-E_{n, \infty, \pm}\left(z_{n}^{ \pm}\right)=\int_{z_{n}^{ \pm}}^{\infty}\left(E_{n, \infty, \pm}\right)^{\prime}(x) \mathrm{d} x=\int_{z_{n}^{ \pm}}^{\infty}\left(-v_{n}^{\prime}\right) v_{n}\left(V_{\infty}-V_{n}\right) \mathrm{d} x \\
& \leq \begin{cases}c \exp \left(-2 \sqrt{\frac{V_{\infty}}{\Lambda}+\xi_{0}}\left(x_{n}+z_{n}^{+}\right)\right) & \left(\text {for } \mathcal{M}_{\lambda, \Lambda}^{+}\right) \\
c \exp \left(-2 \sqrt{\frac{V_{\infty}}{\lambda}+\xi_{0}}\left(x_{n}+z_{n}^{-}\right)\right) & \left(\text {for } \mathcal{M}_{\lambda, \Lambda}^{-}\right)\end{cases} \\
& \leq \begin{cases}c \exp \left(-2 x_{n} \sqrt{\frac{V_{\infty}}{\Lambda}+\xi_{0}}\right) & \left(\text { for } \mathcal{M}_{\lambda, \Lambda}^{+}\right) \\
c \exp \left(-2 x_{n} \sqrt{\frac{V_{\infty}}{\lambda}+\xi_{0}}\right) & \left(\text { for } \mathcal{M}_{\lambda, \Lambda}^{-}\right) .\end{cases}
\end{aligned}
$$

Hence, Step 2 holds.

Step 3: One has

$$
E_{n, \infty, \pm}\left(z_{n}^{ \pm}\right) \leq E_{n, \infty, \pm}\left(y_{n}^{ \pm}\right)
$$

Recalling $y_{n}^{ \pm} \rightarrow-z^{ \pm}$and $x_{n} \rightarrow \infty$, we notice that for each $s \in\left[v_{n}\left(z_{n}^{ \pm}\right), v_{n}(0)\right]$, (V2) and $y_{n}^{ \pm}(s) \leq z_{n}^{ \pm}(s)$ imply $V_{n}\left(y_{n}^{ \pm}(s)\right) \leq V_{n}\left(z_{n}^{ \pm}(s)\right)$. Moreover, we may assume $\varphi_{n} \equiv 0$ in $\left[y_{n}^{ \pm}, \infty\right)$. Hence, noting

$$
\begin{aligned}
& \mathcal{M}_{\lambda, \Lambda}^{ \pm}\left(v_{n}^{\prime \prime}\right)=V_{n} v_{n}-f\left(v_{n}\right) \quad \text { in }\left[y_{n}^{ \pm}, z_{n}^{ \pm}\right], \quad v_{n}^{\prime \prime}(x)<0 \quad \text { in }\left[0, z_{n}^{ \pm}\right), \\
& v_{n}\left(y_{n}^{ \pm}(s)\right)=s=v_{n}\left(z_{n}^{ \pm}(s)\right) \quad \text { for } s \in\left[v_{n}\left(z_{n}^{ \pm}\right), v_{n}(0)\right],
\end{aligned}
$$

we obtain

$$
v_{n}^{\prime \prime}(x)<0 \quad \text { for all } x \in\left(y_{n}^{ \pm}, z_{n}^{ \pm}\right) .
$$


From this it follows that $v_{n}^{\prime}(z)<0<v_{n}^{\prime}(y)$ for $y_{n}^{ \pm} \leq y<0<z \leq z_{n}^{ \pm}$and $y_{n}^{ \pm}, z_{n}^{ \pm} \in C^{1}\left(\left[v_{n}\left(z_{n}^{ \pm}\right), v_{n}(0)\right)\right)$. Thus we see from $(2.20), v_{n}\left(z_{n}^{+}\right)=v_{n}\left(y_{n}^{+}\right)$, the monotonicity of $V_{n}$ and the change of variables $s=v_{n}(x)$ that

$$
\begin{aligned}
E_{n, \infty,+}(0)-E_{n, \infty,+}\left(y_{n}^{+}\right) & =\int_{y_{n}^{+}}^{0}\left(E_{n, \infty,+}\right)^{\prime}(x) \mathrm{d} x \\
& =\int_{y_{n}^{+}}^{0}\left[\frac{\Lambda}{\lambda}\left\{V_{n} v_{n}-f\left(v_{n}\right)\right\}+f\left(v_{n}\right)-V_{\infty} v_{n}\right] v_{n}^{\prime} \mathrm{d} x \\
& =\int_{v_{n}\left(y_{n}^{+}\right)}^{v_{n}(0)}\left[\frac{\Lambda}{\lambda}\left\{V_{n}\left(y_{n}^{+}(s)\right) s-f(s)\right\}+f(s)-V_{\infty} s\right] \mathrm{d} s \\
& \leq \int_{v_{n}\left(z_{n}^{+}\right)}^{v_{n}(0)}\left[\frac{\Lambda}{\lambda}\left\{V_{n}\left(z_{n}^{+}(s)\right) s-f(s)\right\}+f(s)-V_{\infty} s\right] \mathrm{d} s \\
& =-\int_{0}^{z_{n}^{+}}\left[\frac{\Lambda}{\lambda}\left\{V_{n}(x) v_{n}-f\left(v_{n}\right)\right\}+f\left(v_{n}\right)-V_{\infty} v_{n}\right] v_{n}^{\prime} \mathrm{d} x \\
& =-\int_{0}^{z_{n}^{+}}\left(E_{n, \infty,+}\right)^{\prime}(x) \mathrm{d} x=E_{n, \infty,+}(0)-E_{n, \infty,+}\left(z_{n}^{+}\right)
\end{aligned}
$$

Hence, $E_{n, \infty,+}\left(z_{n}^{+}\right) \leq E_{n, \infty,+}\left(y_{n}^{+}\right)$. In a similar way, we can prove $E_{n, \infty,-}\left(z_{n}^{-}\right) \leq$ $E_{n, \infty,-}\left(y_{n}^{-}\right)$and Step 3 holds.

In what follows, we derive the estimates for $E_{n, \infty, \pm}\left(y_{n}^{ \pm}\right)$. First we prove Step 4: $E_{n, \infty, \pm}^{\prime}(x) \leq 0$ in $\left(-\infty, y_{n}^{ \pm}\right)$for sufficiently large $n$.

For $E_{n, \infty,+}$, by $v_{n}^{\prime} \geq 0$ in $\left(-\infty, y_{n}^{+}\right)$and $(2.20)$, if $x<y_{n}^{+}$and $v_{n}^{\prime \prime}(x) \geq 0$, then we have

$$
\left(E_{n, \infty,+}\right)^{\prime}(x)=v_{n}^{\prime}\left\{\left(V_{n}-V_{\infty}\right) v_{n}-s_{n} \varphi_{n}\right\} \leq 0 .
$$

On the other hand, if $x<y_{n}^{+}$and $v_{n}^{\prime \prime}(x)<0$, then $\lambda \leq \Lambda$ gives

$$
\begin{aligned}
\left(E_{n, \infty,+}\right)^{\prime}(x) & =v_{n}^{\prime}\left(\Lambda v_{n}^{\prime \prime}+f\left(v_{n}\right)-V_{\infty} v_{n}\right) \\
& =v_{n}^{\prime}\left\{(\Lambda-\lambda) v_{n}^{\prime \prime}+\left(V_{n}-V_{\infty}\right) v_{n}-s_{n} \varphi_{n}\right\} \leq 0 .
\end{aligned}
$$

Hence, $\left(E_{n, \infty,+}\right)^{\prime}(x) \leq 0$ in $\left(-\infty, y_{n}^{+}\right)$.

For $\mathcal{M}_{\lambda, \Lambda}^{-}$, if $x<y_{n}^{-}$and $v_{n}^{\prime \prime}(x) \geq 0$, then we have

$$
\left(E_{n, \infty,-}\right)^{\prime}(x)=v_{n}^{\prime}\left\{\left(V_{n}-V_{\infty}\right) v_{n}-s_{n} \varphi_{n}\right\} \leq 0 .
$$

On the other hand, we consider the case $v_{n}^{\prime \prime}(x) \leq 0$ and $x<y_{n}^{-}$. We first remark that for sufficiently large $n$, we have $\left(E_{n, \infty,-}\right)^{\prime}(x) \leq 0$ provided $x \in\left(-\infty, 3 \kappa_{0}-x_{n}\right]$ and $v_{n}^{\prime \prime}(x) \leq 0$. In fact, it follows from (2.18), (f2), $\omega_{-}(x) \rightarrow 0$ as $|x| \rightarrow \infty$ and (V1) that one can find $n_{0}$ and $R_{0} \geq 0$ so that

$$
f\left(v_{n}\right)-V_{\infty} v_{n} \leq 0 \quad \text { for each } n \geq n_{0} \quad \text { and } \quad x \leq-R_{0} .
$$

Since we may assume $3 \kappa_{0}-x_{n} \leq-R_{0}$ for $n \geq n_{0}$ due to $x_{n} \rightarrow \infty$, the condition $v_{n}^{\prime \prime}(x) \leq 0$ and $x \leq 3 \kappa_{0}-x_{n}$ give

$$
\left(E_{n, \infty,-}\right)^{\prime}(x)=v_{n}^{\prime}(x)\left\{\lambda v_{n}^{\prime \prime}(x)+f\left(v_{n}\right)-V_{\infty} v_{n}\right\} \leq 0 .
$$


Therefore, we only consider in $\left[3 \kappa_{0}-x_{n}, y_{n}^{-}\right]$and remark that $\varphi_{n} \equiv 0$ on the interval.

Next, we shall show that $f\left(v_{n}(x)\right)-V_{\infty} v_{n}(x) \leq 0$ when $v_{n}^{\prime \prime}(x) \leq 0$ and $x \in\left[3 \kappa_{0}-x_{n}, y_{n}^{-}\right]$. Noting $v_{n}\left(y_{n}^{-}\right)=v_{n}\left(z_{n}^{-}\right), v_{n}(x) \leq v_{n}\left(y_{n}^{-}\right)$for $x \in$ $\left[3 \kappa_{0}-x_{n}, y_{n}^{-}\right]$and

$$
v_{n}^{\prime \prime}\left(z_{n}^{-}\right)=0=V_{n}\left(z_{n}^{-}\right) v_{n}\left(z_{n}^{-}\right)-f\left(v_{n}\left(z_{n}^{-}\right)\right),
$$

we infer from (V2), (f4) and $v_{n}^{\prime}(x) \geq 0$ in $\left[3 \kappa_{0}-x_{n}, y_{n}^{-}\right]$that

$$
0=V_{n}\left(z_{n}^{-}\right)-\frac{f\left(v_{n}\left(y_{n}^{-}\right)\right)}{v_{n}\left(y_{n}^{-}\right)} \leq V_{\infty}-\frac{f\left(v_{n}(x)\right)}{v_{n}(x)} \text { for all } x \in\left[3 \kappa_{0}-x_{n}, y_{n}^{-}\right] .
$$

Thus $f\left(v_{n}(x)\right)-V_{\infty} v_{n}(x) \leq 0$ in $\left[3 \kappa_{0}-x_{n}, y_{n}^{-}\right]$. Therefore, when $x \in$ $\left[3 \kappa_{0}-x_{n}, y_{n}^{-}\right]$and $v_{n}^{\prime \prime}(x) \leq 0$, it follows from $(2.21)$ that

$$
\left(E_{n, \infty,-}\right)^{\prime}(x) \leq \lambda v_{n}^{\prime}(x) v_{n}^{\prime \prime}(x) \leq 0
$$

Hence, Step 4 holds.

Step 5: One has

$$
E_{n, \infty, \pm}\left(y_{n}^{ \pm}\right) \leq \int_{-3 \kappa_{0}-x_{n}}^{-2 \kappa_{0}-x_{n}} v_{n}^{\prime} v_{n}\left(V_{n}-V_{\infty}\right) \mathrm{d} x
$$

By Step 4 , we have $\left(E_{n, \infty, \pm}\right)^{\prime}(x) \leq 0$ in $\left(-\infty, y_{n}^{ \pm}\right)$. Since $E_{n, \infty, \pm}(x) \rightarrow 0$ as $x \rightarrow-\infty$, we obtain

$$
E_{n, \infty, \pm}\left(y_{n}^{ \pm}\right)=\int_{-\infty}^{y_{n}^{ \pm}}\left(E_{n, \infty, \pm}\right)^{\prime}(x) \mathrm{d} x \leq \int_{-3 \kappa_{0}-x_{n}}^{-2 \kappa_{0}-x_{n}}\left(E_{n, \infty, \pm}\right)^{\prime}(x) \mathrm{d} x
$$

Recalling (2.18), (V1), (f2), $x_{n} \rightarrow \infty$ and $\varphi_{n} \equiv 0$ in $\left[-3 \kappa_{0}-x_{n},-2 \kappa_{0}-x_{n}\right]$, we may assume that

$$
\mathcal{M}_{\lambda, \Lambda}^{ \pm}\left(v_{n}^{\prime \prime}\right)=V_{n} v_{n}-f\left(v_{n}\right) \geq 0 \quad \text { in }\left[-3 \kappa_{0}-x_{n},-2 \kappa_{0}-x_{n}\right] .
$$

Hence, $v_{n}^{\prime \prime}(x) \geq 0$ in $\left[-3 \kappa_{0}-x_{n},-2 \kappa_{0}-x_{n}\right]$ and

$$
\left(E_{n, \infty, \pm}\right)^{\prime}(x)=v_{n}^{\prime}\left(V_{n}-V_{\infty}\right) v_{n} \text { in }\left[-3 \kappa_{0}-x_{n},-2 \kappa_{0}-x_{n}\right] .
$$

Thus it is easily seen from (2.22) that Step 5 holds.

Step 6: There exists a $c>0$, which is independent of $n$, such that

$$
\begin{aligned}
& \min \left\{v_{n}(x), v_{n}^{\prime}(x)\right\} \geq c \exp \left(-|x| \sqrt{\frac{V_{\infty}}{\Lambda}+\frac{\xi_{0}}{2(\Lambda+1)}}\right) \quad \text { for } \mathcal{M}_{\lambda, \Lambda}^{+}, \\
& \min \left\{v_{n}(x), v_{n}^{\prime}(x)\right\} \geq c \exp \left(-|x| \sqrt{\frac{V_{\infty}}{\lambda}+\frac{\xi_{0}}{2(\lambda+1)}}\right) \quad \text { for } \mathcal{M}_{\lambda, \Lambda}^{-}
\end{aligned}
$$

for all $x \leq-2 \kappa_{0}-x_{n}$ and sufficiently large $n$.

Set

$$
\psi_{+}(x):=c \exp \left(x \sqrt{\frac{V_{\infty}}{\Lambda}+\frac{\xi_{0}}{2(\Lambda+1)}}\right), \quad \psi_{-}(x):=c \exp \left(x \sqrt{\frac{V_{\infty}}{\lambda}+\frac{\xi_{0}}{2(\lambda+1)}}\right)
$$


where $c>0$ is chosen below. By (2.18), (f2) and $\lambda \leq \Lambda$, we find an $R_{0}>0$ such that

$$
\left|\frac{f\left(v_{n}(x)\right)}{v_{n}(x)}\right| \leq \frac{\lambda}{4(\lambda+1)} \xi_{0} \leq \frac{\Lambda}{4(\Lambda+1)} \xi_{0}
$$

for all $x \leq-R_{0}$ and sufficiently large $n$. Fix a $c>0$ so that $\psi_{ \pm}\left(-R_{0}\right) \leq$ $v_{n}\left(-R_{0}\right)$ for all sufficiently large $n$.

We first notice that

$$
\begin{aligned}
& -\mathcal{M}_{\lambda, \Lambda}^{+}\left(\psi_{+}^{\prime \prime}\right)+\left\{V_{n}+\frac{\Lambda}{4(\Lambda+1)} \xi_{0}\right\} \psi_{+} \\
= & \left\{-V_{\infty}-\frac{\Lambda}{2(\Lambda+1)} \xi_{0}+V_{n}+\frac{\Lambda}{4(\Lambda+1)} \xi_{0}\right\} \psi_{+} \\
= & \left\{V_{n}-V_{\infty}-\frac{\Lambda}{4(\Lambda+1)} \xi_{0}\right\} \psi_{+} \leq 0 \quad \text { in }\left(-\infty,-R_{0}\right) .
\end{aligned}
$$

Similarly,

$$
-\mathcal{M}_{\lambda, \Lambda}^{-}\left(\psi_{-}^{\prime \prime}\right)+\left\{V_{n}+\frac{\lambda}{4(\lambda+1)} \xi_{0}\right\} \psi_{-} \leq 0 \quad \text { in }\left(-\infty,-R_{0}\right) .
$$

On the other hand, for $x \in\left(-\infty,-R_{0}\right)$, it follows from (2.24) that

$$
\begin{aligned}
& 0 \leq s_{n} \varphi_{n}=-\mathcal{M}_{\lambda, \Lambda}^{+}\left(v_{n}^{\prime \prime}\right)+V_{n} v_{n}-f\left(v_{n}\right) \leq-\mathcal{M}_{\lambda, \Lambda}^{+}\left(v_{n}^{\prime \prime}\right)+\left\{V_{n}+\frac{\Lambda}{4(\Lambda+1)} \xi_{0}\right\} v_{n}, \\
& 0 \leq-\mathcal{M}_{\lambda, \Lambda}^{-}\left(v_{n}^{\prime \prime}\right)+\left\{V_{n}+\frac{\lambda}{4(\lambda+1)} \xi_{0}\right\} v_{n}
\end{aligned}
$$

Hence, putting

$$
w_{n,+}(x):=v_{n}-\psi_{+}, \quad w_{n,-}(x):=v_{n}-\psi_{-},
$$

we have

$$
\begin{aligned}
& 0 \leq-\mathcal{M}_{\lambda, \Lambda}^{-}\left(w_{n,+}^{\prime \prime}\right)+\left\{V_{n}+\frac{\Lambda}{4(\Lambda+1)} \xi_{0}\right\} w_{n,+}, \\
& 0 \leq-\mathcal{M}_{\lambda, \Lambda}^{-}\left(w_{n,-}^{\prime \prime}\right)+\left\{V_{n}+\frac{\lambda}{4(\lambda+1)} \xi_{0}\right\} w_{n,-}
\end{aligned}
$$

in $\left(-\infty,-R_{0}\right)$. Recalling $w_{n, \pm}\left(-R_{0}\right) \geq 0$ and $w_{n, \pm} \rightarrow 0$ as $x \rightarrow-\infty, w_{n, \pm}$ do not have negative minima and we get $w_{n, \pm} \geq 0$ in $\left(-\infty,-R_{0}\right]$. Thus (2.23) holds for $v_{n}$.

For $v_{n}^{\prime}$, since $\varphi_{n} \equiv 0$ in $\left(-\infty,-2 \kappa_{0}-x_{n}\right)$, there exists a $c>0$ such that

$$
\mathcal{M}_{\lambda, \Lambda}^{ \pm}\left(v_{n}^{\prime \prime}\right)=V_{n} v_{n}-f\left(v_{n}\right) \geq c v_{n} \quad \text { in }\left(-\infty,-2 \kappa_{0}-x_{n}\right)
$$

for all sufficiently large $n$. Hence, (2.23) holds for $v_{n}^{\prime \prime}$. Noting

$$
v_{n}^{\prime}(x)=\int_{-\infty}^{x} v_{n}^{\prime \prime}(y) \mathrm{d} y,
$$

(2.23) holds.

Step 7: Conclusion (Completion of the proof for (2.16)). 
We first notice that by the choice of $\kappa_{0}>0$, one has

$$
\min _{\left[-3 \kappa_{0}-x_{n},-2 \kappa_{0}-x_{n}\right]}\left(V_{\infty}-V_{n}\right)=\min _{\left[-3 \kappa_{0},-2 \kappa_{0}\right]}\left(V_{\infty}-V(x)\right)>0 .
$$

By Step 6, we observe that for $x \in\left[-3 \kappa_{0}-x_{n},-2 \kappa_{0}-x_{n}\right]$

$$
v_{n}^{\prime}(x) v_{n}(x) \geq\left\{\begin{array}{lc}
c \exp \left(-2\left(3 \kappa_{0}+x_{n}\right) \sqrt{\left.\frac{V_{\infty}}{\Lambda}+\frac{\xi_{0}}{2(\Lambda+1)}\right)}\right. & \left(\text { for } \mathcal{M}_{\lambda, \Lambda}^{+}\right), \\
c \exp \left(-2\left(3 \kappa_{0}+x_{n}\right) \sqrt{\left.\frac{V_{\infty}}{\lambda}+\frac{\xi_{0}}{2(\lambda+1)}\right)}\right. & \left(\text { for } \mathcal{M}_{\lambda, \Lambda}^{-}\right) .
\end{array}\right.
$$

Therefore, using (2.25), (2.26) and Step 5, we obtain

$$
\begin{array}{ll}
-E_{n, \infty,+}\left(y_{n}^{+}\right) \geq c \exp \left(-2 x_{n} \sqrt{\frac{V_{\infty}}{\Lambda}+\frac{\xi_{0}}{2(\Lambda+1)}}\right) \quad\left(\text { for } \mathcal{M}_{\lambda, \Lambda}^{+}\right), \\
-E_{n, \infty,-}\left(y_{n}^{-}\right) \geq c \exp \left(-2 x_{n} \sqrt{\frac{V_{\infty}}{\lambda}+\frac{\xi_{0}}{2(\lambda+1)}}\right) \quad\left(\text { for } \mathcal{M}_{\lambda, \Lambda}^{-}\right)
\end{array}
$$

for some $c>0$. However, by Steps 2 and 3, we have a contradiction. Hence, we may find an $M_{2}>0$ so that $x_{n} \leq M_{2}$.

For the lower bound of $\left(x_{n}\right)$, by introducing $\tilde{u}_{n}(x):=u_{n}(-x)$, we can reduce the case into the case $x_{n} \rightarrow \infty$. Thus (2.16) holds.

We finally derive a contradiction in order to complete the proof of Proposition 2.9. By (2.16), we may assume $x_{n} \rightarrow x_{0}$. Next, from Lemmas 2.6 and 2.8 , we observe that if $s_{n} \geq \tilde{t}\left(f, V_{\infty}\right)$, then

$$
\frac{\kappa_{0}^{2}}{4 \Lambda} s_{n} \leq\left\|u_{n}\right\|_{L^{\infty}(\mathbf{R})} \leq M_{0} .
$$

Therefore, $\left(s_{n}\right)$ is also bounded and assume that $s_{n} \rightarrow s_{0}$. Thus from the equation, we also get $u_{n} \rightarrow u_{0}$ in $C_{\text {loc }}^{2}(\mathbf{R})$,

$$
\begin{aligned}
& -\mathcal{M}_{\lambda, \Lambda}^{ \pm}\left(u_{0}^{\prime \prime}\right)+V u_{0}=f\left(u_{0}\right)+s_{0} \varphi_{0} \quad \text { in } \mathbf{R}, \quad u_{0}\left(x_{0}\right)=\max _{\mathbf{R}} u_{0}, \\
& u_{0}^{\prime}(y) \leq 0 \leq u_{0}^{\prime}(x) \quad \text { for } x \leq x_{0} \leq y, \quad u_{0} \geq 0 \text { in } \mathbf{R} .
\end{aligned}
$$

If $u_{0}\left(x_{0}\right)=0$, namely $u_{0} \equiv 0$, then by the monotonicity of $u_{n}\left(u_{n}^{\prime}(y) \leq 0 \leq\right.$ $u_{n}^{\prime}(x)$ for $\left.x \leq x_{n} \leq y\right)$, we choose an $R_{0}>3 \kappa_{0}$ so that

$$
V u_{n}-f\left(u_{n}\right) \geq \frac{V_{0}}{2} u_{n}
$$

for all $|x| \geq R_{0}$ and sufficiently large $n$. Therefore, we have

$$
\mathcal{M}_{\lambda, \Lambda}^{ \pm}\left(u_{n}^{\prime \prime}\right)=V u_{n}-f\left(u_{n}\right) \geq \frac{V_{0}}{2} u_{n} \quad \text { for every }|x| \geq R_{0} .
$$

Hence, we may derive the uniform exponential decay:

$$
u_{n}(x) \leq c \exp \left(-|x| \sqrt{\frac{V_{0}}{2 \Lambda}}\right)
$$


for all $x \in \mathbf{R}$ and $n$. By the definition of $X_{\eta_{1}}$ and (2.3), this asserts that $\left(u_{n}\right)$ is bounded in $X_{\eta_{1}}$, however, this contradicts $\left\|u_{n}\right\|_{X_{\eta_{1}}} \rightarrow \infty$.

Next we consider the case $u_{0}\left(x_{0}\right)>0$ and shall show that $\lim _{|x| \rightarrow \infty} u_{0}(x)=$ 0 . If this is true, then as in the above, we can derive a uniform exponential decay and get a contradiction. Set $u_{\infty}:=\lim _{x \rightarrow \infty} u_{0}(x)$. Since $u_{0}$ is a bounded solution of (2.27), we have

$$
\lim _{x \rightarrow \infty} \mathcal{M}_{\lambda, \Lambda}^{ \pm}\left(u_{0}^{\prime \prime}\right)(x)=\lim _{x \rightarrow \infty}\left(V u_{0}-f\left(u_{0}\right)-s_{0} \varphi_{0}\right)=V_{\infty} u_{\infty}-f\left(u_{\infty}\right) .
$$

Thus by (2.15), either $u_{\infty}=0$ or else $u_{\infty}=s_{\infty}$. Let us assume $u_{\infty}=s_{\infty}$. From (2.27), we get $u_{0} \geq s_{\infty}$ in $\left[x_{0}, \infty\right)$. Since $V_{\infty} s-f(s)<0$ for $s>s_{\infty}$, one sees that

$\mathcal{M}_{\lambda, \Lambda}^{ \pm}\left(u_{0}^{\prime \prime}\right)=V u_{0}-f\left(u_{0}\right)-s_{0} \varphi_{0} \leq V_{\infty} u_{0}-f\left(u_{0}\right)-s_{0} \varphi_{0} \leq 0 \quad$ in $\left(x_{0}, \infty\right)$.

Since $u_{0}^{\prime}\left(x_{0}\right)=0$ and $u_{0}(x) \rightarrow s_{\infty}$ as $x \rightarrow \infty$, we conclude that $V \equiv V_{\infty}$, $u_{0} \equiv s_{\infty}$ and $s_{0} \varphi_{0} \equiv 0$ in $\left[x_{0}, \infty\right)$. Hence, by $(2.9)$ and $V \equiv V_{\infty}$ in $\left[x_{0}, \infty\right)$, we see $3 \kappa_{0} \leq x_{0}$. Thus we may assume $2 \kappa_{0} \leq x_{n}$. Now set

$$
\begin{aligned}
& E_{n,+}(x):=\frac{\Lambda}{2}\left(u_{n}^{\prime}(x)\right)^{2}+F\left(u_{n}(x)\right)-\frac{V(x)}{2} u_{n}^{2}(x) \text { for } \mathcal{M}_{\lambda, \Lambda}^{+}, \\
& E_{n,-}(x):=\frac{\lambda}{2}\left(u_{n}^{\prime}(x)\right)^{2}+F\left(u_{n}(x)\right)-\frac{V(x)}{2} u_{n}^{2}(x) \text { for } \mathcal{M}_{\lambda, \Lambda}^{-} .
\end{aligned}
$$

Since $x_{n} \geq 2 \kappa_{0}, V^{\prime}(x) \geq 0$ in $\left[x_{n}, \infty\right)$ and $u_{n}(x) \rightarrow 0$ as $|x| \rightarrow \infty$, arguing for the case $v_{n}$ in the above, we may find unique $z_{n}^{ \pm} \geq x_{n}$ such that

$$
u_{n}^{\prime \prime}(x)<0=u_{n}^{\prime \prime}\left(z_{n}^{ \pm}\right)<u_{n}^{\prime \prime}(y) \text { for } x_{n} \leq x<z_{n}^{ \pm}<y .
$$

Therefore,

$$
\left(E_{n, \pm}\right)^{\prime}(x)=-\frac{V^{\prime}}{2} u_{n}^{2} \leq 0 \quad \text { in }\left[z_{n}^{ \pm}, \infty\right), \quad \lim _{x \rightarrow \infty} E_{n, \pm}(x)=0, \quad E_{n, \pm}\left(z_{n}^{ \pm}\right) \geq 0 .
$$

By $u_{n}^{\prime \prime}\left(z_{n}^{ \pm}\right)=0$, one has

$$
V_{0} \leq V\left(z_{n}^{ \pm}\right)=\frac{f\left(u_{n}\left(z_{n}^{ \pm}\right)\right)}{u_{n}\left(z_{n}^{ \pm}\right)} .
$$

From (f2), we may find a $\delta_{0}>0$ so that $\delta_{0} \leq u_{n}\left(z_{n}^{ \pm}\right)$. Recalling $x_{0} \leq$ $\liminf _{n \rightarrow \infty} z_{n}^{ \pm}, V \equiv V_{\infty}$ in $\left[x_{0}, \infty\right)$ and (2.15), we obtain

$$
\begin{aligned}
& u_{n}\left(z_{n}^{ \pm}\right) \rightarrow s_{\infty}, \\
& F\left(u_{n}\left(z_{n}^{ \pm}\right)\right)-\frac{V\left(z_{n}^{ \pm}\right)}{2} u_{n}\left(z_{n}^{ \pm}\right)^{2} \rightarrow F\left(s_{\infty}\right)-\frac{V_{\infty}}{2} s_{\infty}^{2}=G_{\infty}\left(s_{\infty}\right)<0 .
\end{aligned}
$$

Combining with $E_{n, \pm}\left(z_{n}^{ \pm}\right) \geq 0$, we may find a $\delta_{1}>0$ so that

$$
\left(u_{n}^{\prime}\left(z_{n}^{ \pm}\right)\right)^{2} \geq \delta_{1} \text {. }
$$

Noting that $u_{n}^{\prime}\left(x_{n}\right)=0$ and $\left(u_{n}^{\prime \prime}\right)$ is bounded in $L^{\infty}(\mathbf{R})$, we have $0<\delta_{2} \leq$ $z_{n}^{ \pm}-x_{n}$ for some $\delta_{2}>0$, and

$$
\left(u_{n}^{\prime}(x)\right)^{2} \geq \delta_{3}^{2}>0 \quad \text { in }\left[z_{n}^{ \pm}-\delta_{4}, z_{n}^{ \pm}\right]
$$


for some $\delta_{3}, \delta_{4}>0$ with $\delta_{4} \leq \delta_{2}$. Thus

$$
u_{n}\left(x_{n}\right) \geq u_{n}\left(z_{n}^{ \pm}-\delta_{4}\right) \geq u_{n}\left(z_{n}^{ \pm}\right)+\delta_{3} \delta_{4} \rightarrow s_{\infty}+\delta_{3} \delta_{4} .
$$

This contradicts $u_{n}\left(x_{n}\right) \rightarrow u_{0}\left(x_{0}\right)=s_{\infty}$. Hence, $u_{\infty}=0$.

For $\lim _{x \rightarrow-\infty} u_{0}(x)=0$, by introducing $v_{n}(x)=u_{n}(-x)$ and $v_{0}(x)=$ $u_{0}(-x)$, we can reduce into the former case and get $\lim _{x \rightarrow-\infty} u_{0}(x)=0$. Now we complete the proof of Proposition 2.9.

\section{Non-EXistence THEOREM}

In this section we prove Theorem 1.2 that asserts that the equation (1.1) does not have a solution when $V$ is monotone. The argument below is similar to that of Proposition 2.9.

Proof of Theorem 1.2. Let us suppose for contradiction that $u$ is a positive solution of (1.1) and let $x_{0}$ be a maximum point of $u$. Noting that $V$ is non-decreasing and that the argument in Lemma 2.7 works under (f1) and (1.3), if $\bar{x}$ satisfies $u^{\prime}(\bar{x})=0$ then $u^{\prime}(x)<0$ for all $x \in(\bar{x}, \infty)$. Thus $x_{0}$ is the unique critical point of $u$.

To proceed further, we make some preparations. Since $u$ is strictly decreasing in $\left(x_{0}, \infty\right)$ and $V$ non-decreasing in $\mathbf{R}$, by (f4), we see that the function

$$
h(x):=V(x)-\frac{f(u(x))}{u(x)}:\left[x_{0}, \infty\right) \rightarrow \mathbf{R}
$$

is strictly increasing. Moreover, since $u^{\prime \prime}\left(x_{0}\right) \leq 0$ and $\mathcal{M}_{\lambda, \Lambda}^{ \pm}\left(u^{\prime \prime}\right)=u h(x)$ in $\left[x_{0}, \infty\right)$, we have $h\left(x_{0}\right) \leq 0$. Hence, by $h(x) \rightarrow \bar{V}>0$ as $x \rightarrow \infty$ thanks to (V2'), there is a unique $z^{ \pm} \geq x_{0}$ such that $h\left(z^{ \pm}\right)=0$. In particular, $u^{\prime \prime}(y)>0=u^{\prime \prime}\left(z^{ \pm}\right)>u^{\prime \prime}(x)$ for all $x_{0} \leq x<z^{ \pm}<y$. Recalling that $u$ is strictly increasing in $\left(-\infty, x_{0}\right)$ and decreasing in $\left(x_{0}, \infty\right), u$ has two inverse functions $y^{ \pm}(s)$ and $z^{ \pm}(s)$ satisfying $y^{ \pm}(s)<x_{0}<z^{ \pm}(s)$ for $0<s<u\left(x_{0}\right)$. Next we define $y^{ \pm}=y^{ \pm}\left(u\left(z^{ \pm}\right)\right)$and

$$
\begin{array}{ll}
H_{+}(x)=\frac{\Lambda}{2}\left(u^{\prime}(x)\right)^{2}+F(u(x))-\frac{V\left(z^{+}\right)}{2} u^{2}(x) & \text { for } \mathcal{M}_{\lambda, \Lambda}^{+}, \\
H_{-}(x)=\frac{\lambda}{2}\left(u^{\prime}(x)\right)^{2}+F(u(x))-\frac{V\left(z^{-}\right)}{2} u^{2}(x) & \text { for } \mathcal{M}_{\lambda, \Lambda}^{-} .
\end{array}
$$

To complete the proof we proceed in various steps.

Step 1: $H_{ \pm}\left(z^{ \pm}\right) \geq 0$ and if $V\left(z^{ \pm}\right)<\bar{V}$, then $H_{ \pm}\left(z^{ \pm}\right)>0$.

We start with

$$
\begin{aligned}
& H_{ \pm}^{\prime}(x)=u^{\prime}(x)\left(V(x)-V\left(z^{ \pm}\right)\right) u(x) \text { if } u^{\prime \prime}(x) \geq 0 \\
& H_{+}^{\prime}(x)=u^{\prime}(x)\left(\frac{\Lambda}{\lambda} h(x) u(x)+f(u(x))-V\left(z^{+}\right) u(x)\right) \text { if } u^{\prime \prime}(x) \leq 0 \\
& H_{-}^{\prime}(x)=u^{\prime}(x)\left(\frac{\lambda}{\Lambda} h(x) u(x)+f(u(x))-V\left(z^{-}\right) u(x)\right) \text { if } u^{\prime \prime}(x) \leq 0 .
\end{aligned}
$$


Noticing that $u^{\prime}<0 \leq V^{\prime}$ and $0 \leq u^{\prime \prime}$ in $\left(z^{ \pm}, \infty\right)$, from (3.1) we have $H_{ \pm}^{\prime}(x) \leq 0$ in $\left(z^{ \pm}, \infty\right)$. In case $V\left(z^{ \pm}\right)<\bar{V}$ we additionally have $H_{ \pm}^{\prime}(x) \not \equiv 0$. From $H_{ \pm}(x) \rightarrow 0$ as $|x| \rightarrow \infty$ we conclude that Step 1 holds.

Step 2: $H_{ \pm}\left(z^{ \pm}\right) \leq H_{ \pm}\left(y^{ \pm}\right)$and if $V \not \equiv$ const. in $\left[y^{ \pm}, z^{ \pm}\right]$and $y^{ \pm}<z^{ \pm}$, then $H_{ \pm}\left(z^{ \pm}\right)<H_{ \pm}\left(y^{ \pm}\right)$.

When $z^{ \pm}=x_{0}$ then $z^{ \pm}=y^{ \pm}$and the conclusion clearly holds. In case $x_{0}<z^{ \pm}$we use arguments similar to those of Step 3 of the proof of Proposition 2.9. Since $u^{\prime \prime}\left(z^{ \pm}(s)\right)<0$ for every $s \in\left(u\left(z^{ \pm}\right), u\left(x_{0}\right)\right)$, $u^{\prime \prime}\left(z^{ \pm}\right)=0=h\left(z^{ \pm}\right)$and $V$ is non-decreasing, we observe that for each $s \in\left(u\left(z^{ \pm}\right), u\left(x_{0}\right)\right)$,

$$
h\left(y^{ \pm}(s)\right)=V\left(y^{ \pm}(s)\right)-\frac{f(s)}{s} \leq V\left(z^{ \pm}(s)\right)-\frac{f(s)}{s}=h\left(z^{ \pm}(s)\right)<h\left(z^{ \pm}\right)=0 .
$$

From $\mathcal{M}_{\lambda, \Lambda}^{ \pm}\left(u^{\prime \prime}\right)=u(x) h(x)$ it follows that $u^{\prime \prime}(x)<0$ in $\left(y^{ \pm}, z^{ \pm}\right)$. Hence, by (3.2), (3.4) and changing variables $s=u(x)$, we have

$$
\begin{aligned}
H_{+}\left(x_{0}\right)-H_{+}\left(y^{+}\right) & =\int_{y^{+}}^{x_{0}}\left[\frac{\Lambda}{\lambda} h(x) u(x)+f(u(x))-V\left(z^{+}\right) u(x)\right] u^{\prime}(x) \mathrm{d} x \\
& =\int_{u\left(y^{+}\right)}^{u\left(x_{0}\right)}\left[\frac{\Lambda}{\lambda} h\left(y^{+}(s)\right) s+f(s)-V\left(z^{+}\right) s\right] \mathrm{d} s \\
& \leq \int_{u\left(z^{+}\right)}^{u\left(x_{0}\right)}\left[\frac{\Lambda}{\lambda} h\left(z^{+}(s)\right) s+f(s)-V\left(z^{+}\right) s\right] \mathrm{d} s \\
& =-\int_{x_{0}}^{z^{+}}\left[\frac{\Lambda}{\lambda} h(x) u(x)+f(u(x))-V\left(z^{+}\right) u(x)\right] u^{\prime}(x) \mathrm{d} x \\
& =-\int_{x_{0}}^{z^{+}} H_{+}^{\prime}(x) \mathrm{d} x=H_{+}\left(x_{0}\right)-H_{+}\left(z^{+}\right) .
\end{aligned}
$$

Thus $H_{+}\left(z^{+}\right) \leq H_{+}\left(y^{+}\right)$and if $V \not \equiv$ const. in $\left[y^{+}, z^{+}\right]$, then we have $V\left(y^{+}\right)<$ $V\left(z^{+}\right)$and $h\left(y^{ \pm}(s)\right)<h\left(z^{ \pm}(s)\right)$ for $s$ close to $u\left(z^{ \pm}\right)$. Hence, in this case, $H_{+}\left(z^{+}\right)<H_{+}\left(y^{+}\right)$holds. Using (3.3) instead of (3.2), the case of $H^{-}$is treated in a similar way.

Step 3: $H_{ \pm}^{\prime}(x) \leq 0$ in $\left(-\infty, y^{ \pm}\right)$and if $V \not \equiv$ const. in $\left(-\infty, y^{ \pm}\right)$, then $H_{ \pm}^{\prime} \not \equiv 0$.

First we consider $H_{+}$. We observe that $u^{\prime}>0$ and $V^{\prime}(x) \geq 0$ in $\left(-\infty, y^{+}\right)$, so that when $u^{\prime \prime}(x) \geq 0,(3.1)$ implies

$$
H_{+}^{\prime}(x)=u^{\prime}(x)\left(V(x)-V\left(z^{+}\right)\right) u(x) \leq 0 .
$$

On the other hand, if $u^{\prime \prime}(x)<0$, then recalling that $\lambda \leq \Lambda$, we have

$$
\begin{aligned}
H_{+}^{\prime}(x) & =u^{\prime}\left(\Lambda u^{\prime \prime}+f(u)-V\left(z^{ \pm}\right) u\right) \\
& =u^{\prime}\left\{(\Lambda-\lambda) u^{\prime \prime}+\left(V(x)-V\left(z^{+}\right)\right) u\right\} \leq 0 .
\end{aligned}
$$


Hence, $H_{+}^{\prime} \leq 0$ in $\left(-\infty, y^{+}\right)$. If $V \not \equiv$ const. in $\left(-\infty, y^{+}\right)$, we may find $x_{1}<y^{+}$such that $V\left(x_{1}\right)<V\left(y^{+}\right) \leq V\left(z^{+}\right)$. Then, from (3.5) or (3.6), we have $H_{+}^{\prime}\left(x_{1}\right)<0$.

Next we consider $H_{-}$. We have $u^{\prime}>0$ for $x<y^{-}$, hence if $u^{\prime \prime}(x) \geq 0$, then we have

$$
H_{-}^{\prime}(x)=u^{\prime}(x)\left\{V(x)-V\left(z^{-}\right)\right\} u(x) \leq 0 .
$$

On the other hand, assume that $u^{\prime \prime}(x)<0$. Since $u^{\prime}>0$ in $\left(-\infty, y^{-}\right)$, we get $u(x)<u\left(y^{-}\right)=u\left(z^{-}\right)$. Therefore, by the definition of $z^{-}$and (f4), we find

$$
0=h\left(z^{-}\right)=V\left(z^{-}\right)-\frac{f\left(u\left(z^{-}\right)\right)}{u\left(z^{-}\right)}<V\left(z^{-}\right)-\frac{f(u(x))}{u(x)},
$$

which yields $f(u(x))-V\left(z^{-}\right) u(x)<0$. Thus, from (3.3) and monotonicity of $V$, it follows that

$$
\begin{aligned}
H_{-}^{\prime}(x)= & u^{\prime}(x)\left[\frac{\lambda}{\Lambda} h(x) u(x)+f(u(x))-V\left(z^{-}\right) u(x)\right] \\
= & u^{\prime}(x)\left[\frac{\lambda}{\Lambda}\left\{V(x)-V\left(z^{-}\right)\right\} u(x)\right. \\
& \left.+\left(1-\frac{\lambda}{\Lambda}\right)\left\{f(u(x))-V\left(z^{-}\right) u(x)\right\}\right] \leq 0 .
\end{aligned}
$$

By (3.7) and (3.8), we get $H_{-}^{\prime}(x) \leq 0$ in $\left(-\infty, y^{-}\right)$. Moreover, it is easily seen that when $V \not \equiv$ const. in $\left(-\infty, y^{-}\right), H_{-}^{\prime} \not \equiv 0$ holds.

Step 4: Conclusion.

By Steps 1-3, we get

$$
0 \leq H_{ \pm}\left(z^{ \pm}\right) \leq H_{ \pm}\left(y^{ \pm}\right)=\int_{-\infty}^{y^{ \pm}} H_{ \pm}^{\prime}(x) \mathrm{d} x \leq 0 .
$$

However, $\underline{V}<\bar{V}$, so we have $V \not \equiv$ const. in either $\left(-\infty, y^{ \pm}\right)$or $\left(y^{ \pm}, z^{ \pm}\right)$or $\left(z^{ \pm}, \infty\right)$. Consequently, at least one inequality in (3.9) is strict, providing a contradiction and completing the proof.

Acknowledgements: The authors would like to thank Lawrence Evans for pointing up the variational structure of (1.1). P.F. was partially supported BASAL-CMM projects. N.I. was partially supported by JSPS Research Fellowships 24-2259 and JSPS KAKENHI Grant Number JP16K17623. The second author would like to thank Universidad de Chile, where this work was started, for their hospitality.

\section{Appendix A. Proof of Proposition 2.1}

Here we prove Proposition 2.1. 
Proof of Proposition 2.1. We first prove the existence of solutions. For $\alpha>$ 0 , we consider

$$
\begin{aligned}
& -u^{\prime \prime}=\Lambda^{-1} g_{\infty}(u) \quad \text { in } \mathbf{R}, \quad\left(u^{\prime}(0), u(0)\right)=(0, \alpha), \\
& -u^{\prime \prime}=\lambda^{-1} g_{\infty}(u) \quad \text { in } \mathbf{R}, \quad\left(u^{\prime}(0), u(0)\right)=(0, \alpha)
\end{aligned}
$$

where $g_{\infty}(s):=f(s)-V_{\infty} s$, and we write $u_{\Lambda, \alpha}$ and $u_{\lambda, \alpha}$ for unique solutions of (A.1) and (A.2). By (f1)-(f4), it is well known that there exists an $\alpha_{0}>0$ so that $u_{\Lambda, \alpha}(x)$ hits zero at some point $x_{\alpha}>0\left(u_{\Lambda, \alpha}\left(x_{\alpha}\right)=0\right)$ if $\alpha>\alpha_{0}$, $u_{\Lambda, \alpha_{0}}$ is a positive solution of (A.1) and $u_{\Lambda, \alpha_{0}}(x) \rightarrow 0$ as $|x| \rightarrow \infty$, and $u_{\Lambda, \alpha}(x)$ a positive periodic solution of (A.1) when $\alpha<\alpha_{0}$. The number $\alpha_{0}>0$ is characterized by

$$
G_{\infty}\left(\alpha_{0}\right)=0
$$

and (A.3) has a unique positive solution due to (2.15) (or (f1)-(f4)). For instance, see $[2,9]$. Therefore, (A.3) yields $\alpha_{0}>s_{\infty}$. The same statement holds for $u_{\lambda, \alpha}$.

For $\mu>0$, set

$$
E[u, \mu](x):=\frac{1}{2}\left(u^{\prime}(x)\right)^{2}+\mu^{-1} G_{\infty}(u(x)) .
$$

Then it is easily seen that

$$
\frac{\mathrm{d}}{\mathrm{d} x} E\left[u_{\Lambda, \alpha}, \Lambda\right](x) \equiv 0 \equiv \frac{\mathrm{d}}{\mathrm{d} x} E\left[u_{\lambda, \alpha}, \lambda\right](x) \quad \text { in } \mathbf{R} .
$$

In particular, since $u_{\Lambda, \alpha_{0}}(x), u_{\Lambda, \alpha_{0}}^{\prime}(x), u_{\lambda, \alpha_{0}}(x), u_{\lambda, \alpha_{0}}^{\prime}(x) \rightarrow 0$ as $x \rightarrow \infty$, we have $E\left[u_{\Lambda, \alpha_{0}}, \Lambda\right] \equiv 0 \equiv E\left[u_{\lambda, \alpha_{0}}, \lambda\right]$ in $\mathbf{R}$.

Since $u_{\Lambda, \alpha_{0}}(0)=\alpha_{0}>s_{\infty}$ and $u_{\Lambda, \alpha_{0}}(x) \rightarrow 0$ as $x \rightarrow \infty$, we may choose $x_{\Lambda}>0$ so that $u_{\Lambda, \alpha_{0}}\left(x_{\Lambda}\right)=s_{\infty}$ and $u_{\Lambda, \alpha_{0}}(x)<s_{\infty}$ for every $x>x_{\Lambda}$. Recalling $G_{\infty}\left(s_{\infty}\right)<0$ and $E\left[u_{\Lambda, \alpha_{0}}, \Lambda\right]\left(x_{\Lambda}\right)=0$, we obtain $u_{\Lambda, \alpha_{0}}^{\prime}\left(x_{\Lambda}\right)<0$ and

$$
\begin{aligned}
\lambda^{-1} \min _{\mathbf{R}} G_{\infty} & <\frac{1}{2}\left(u_{\Lambda, \alpha_{0}}^{\prime}\left(x_{\Lambda}\right)\right)^{2}+\lambda^{-1} G_{\infty}\left(u_{\Lambda, \alpha_{0}}\left(x_{\Lambda}\right)\right) \\
& =E\left[u_{\Lambda, \alpha_{0}}, \lambda\right]\left(x_{\Lambda}\right)<E\left[u_{\Lambda, \alpha_{0}}, \Lambda\right]\left(x_{\Lambda}\right)=0 .
\end{aligned}
$$

By (A.3) and (2.15), the equation

$$
\lambda^{-1} G_{\infty}(s)=E\left[u_{\Lambda, \alpha_{0}}, \lambda\right]\left(x_{\Lambda}\right) \in\left(\lambda^{-1} \min _{\mathbf{R}} G_{\infty}, 0\right)
$$

has two solutions $0<s_{1}<s_{\infty}<s_{2}<\alpha_{0}$.

Now we consider $u_{\lambda, s_{2}}(x)$. Since $u_{\lambda, s_{2}}$ is periodic and $E\left[u_{\lambda, s_{2}}, \lambda\right](x)=$ $E\left[u_{\lambda, s_{2}}, \lambda\right](0)=\lambda^{-1} G_{\infty}\left(s_{2}\right)<0$ in $\mathbf{R}$, we observe that

$$
\max _{\mathbf{R}} u_{\lambda, s_{2}}=s_{2}>s_{\infty}>s_{1}=\min _{\mathbf{R}} u_{\lambda, s_{2}} \text {. }
$$

Hence, we may select a $y_{1}>0$ so that $u_{\lambda, s_{2}}(x)>s_{\infty}=u_{\lambda, s_{2}}\left(y_{1}\right)$ for each $x \in\left[0, y_{1}\right)$. From the choice of $y_{1}$ and (A.4), it follows that

$$
u_{\lambda, s_{2}}\left(y_{1}\right)=s_{\infty}=u_{\Lambda, \alpha_{0}}\left(x_{\Lambda}\right), \quad E\left[u_{\lambda, s_{2}}, \lambda\right]\left(y_{1}\right)=\lambda^{-1} G_{\infty}\left(s_{2}\right)=E\left[u_{\Lambda, \alpha_{0}}, \lambda\right]\left(x_{\Lambda}\right),
$$


which implies $\left|u_{\lambda, s_{2}}^{\prime}\left(y_{1}\right)\right|=\left|u_{\Lambda, \alpha_{0}}^{\prime}\left(x_{\Lambda}\right)\right|$. By $u_{\lambda, s_{2}}^{\prime}\left(y_{1}\right), u_{\Lambda, \alpha_{0}}^{\prime}\left(x_{\Lambda}\right) \leq 0$ due to the definition of $y_{1}$ and $x_{\Lambda}$, we obtain $u_{\lambda, s_{2}}^{\prime}\left(y_{1}\right)=u_{\Lambda, \alpha_{0}}^{\prime}\left(x_{\Lambda}\right)$. Thus, set

$$
u(x):= \begin{cases}u_{\lambda, s_{2}}(x) & \text { if } 0 \leq x \leq y_{1}, \\ u_{\Lambda, \alpha_{0}}\left(x-y_{1}+x_{\Lambda}\right) & \text { if } y_{1}<x .\end{cases}
$$

and $u(x):=u(-x)$ for $x<0$, it is easily seen that $u \in C^{1}(\mathbf{R})$ and $u$ satisfies $-\mathcal{M}_{\lambda, \Lambda}^{+}\left(u^{\prime \prime}\right)=g_{\infty}(u)$ in $\mathbf{R} \backslash\left\{ \pm y_{1}\right\}$. In addition, from the definition of $u$, it follows that

$$
\lim _{h \downarrow 0} \frac{u^{\prime}\left(y_{1}+h\right)-u^{\prime}\left(y_{1}\right)}{h}=0=\lim _{h \uparrow 0} \frac{u^{\prime}\left(y_{1}+h\right)-u^{\prime}\left(y_{1}\right)}{h} .
$$

Hence, $u \in C^{2}(\mathbf{R})$ and $u$ is a solution of (2.1). Moreover, we observe that $u^{\prime \prime}(x)<0<u^{\prime \prime}(y)$ for all $|x|<y_{1}$ and $|y|>y_{1}$. Further, it is known that $u_{\Lambda, \alpha_{0}}$ decays exponentially, so does $u$.

For (2.2), we start with $u_{\lambda, \alpha_{0}}$ instead of $u_{\Lambda, \alpha_{0}}$. Then we choose an $x_{\lambda}>0$ so that $u_{\lambda, \alpha_{0}}(x)<s_{\infty}=u_{\lambda, \alpha_{0}}\left(x_{\lambda}\right)$ for every $x \in\left(x_{\lambda}, \infty\right)$. In this case, instead of (A.4), we consider the equation

$$
\Lambda^{-1} G_{\infty}(s)=E\left[u_{\lambda, \alpha_{0}}, \Lambda\right]\left(x_{\lambda}\right)>E\left[u_{\lambda, \alpha_{0}}, \lambda\right]\left(x_{\lambda}\right)=0
$$

and this equation has only one solution $s_{1}>\alpha_{0}$ due to (2.15). Let us consider $u_{\Lambda, s_{1}}$. By $s_{1}>\alpha_{0}$, we may find a $z_{1}>0$ satisfying $u_{\Lambda, s_{1}}\left(z_{1}\right)=0$. Thus, choose a $y_{1}>0$ so that $u_{\Lambda, s_{1}}(x)>s_{\infty}=u_{\Lambda, s_{1}}\left(y_{1}\right)$ for all $x \in\left[0, y_{1}\right)$ and set

$$
u(x):= \begin{cases}u_{\Lambda, s_{1}}(x) & \text { if } 0 \leq x \leq y_{1}, \\ u_{\lambda, \alpha_{0}}\left(x-y_{1}+x_{\lambda}\right) & \text { if } y_{1}<x .\end{cases}
$$

Then as in the above, we can check that $u \in C^{2}(\mathbf{R})$ is a solution of (2.2), decays exponentially and $u^{\prime \prime}(x)<0<u^{\prime \prime}(y)$ for each $|x|<y_{1}$ and $|y|>y_{1}$.

Next, we prove the uniqueness of solutions of (2.1) and (2.2). Let $u_{1}$ be a solution of (2.1) constructed in the above and $u$ any solution of (2.1). By the sign property of $g_{\infty}$, we deduce that $u(0) \geq s_{\infty}$. Moreover, notice that

$$
-\mathcal{M}_{\lambda, \Lambda}^{+}\left(u^{\prime \prime}\right)=g_{\infty}(u) \quad \text { in } \mathbf{R} \quad \Leftrightarrow \quad-u^{\prime \prime}=\left(\mathcal{M}_{\lambda, \Lambda}^{+}\right)^{-1}\left(g_{\infty}(u)\right) \quad \text { in } \mathbf{R}
$$

where $\left(\mathcal{M}_{\lambda, \Lambda}^{+}\right)^{-1}(s)=\Lambda^{-1} s$ if $s \geq 0$ and $\left(\mathcal{M}_{\lambda, \Lambda}^{+}\right)^{-1}(s)=\lambda^{-1} s$ if $s<0$. Since $\left(\mathcal{M}_{\lambda, \Lambda}^{+}\right)^{-1}$ and $f$ are locally Lipschitz continuous, the initial value problem

$$
-u^{\prime \prime}=\left(\mathcal{M}_{\lambda, \Lambda}^{+}\right)^{-1}\left(g_{\infty}(u)\right) \quad \text { in } \mathbf{R}, \quad\left(u^{\prime}(z), u(z)\right)=\left(\alpha_{1}, \alpha_{2}\right)
$$

has a unique solution $u_{z, \alpha_{1}, \alpha_{2}}$ for every $z, \alpha_{1} \in \mathbf{R}$ and $\alpha_{2}>0$. Since $g_{\infty}\left(s_{\infty}\right)=0$, if $u(0)=s_{\infty}$, then we infer that $u \equiv u_{0,0, s_{\infty}} \equiv s_{\infty}$, which contradicts $u(x) \rightarrow 0$ as $x \rightarrow \infty$. Hence, $u(0)>s_{\infty}$.

Now choose $z_{\Lambda}>0$ so that $u\left(z_{\Lambda}\right)=s_{\infty}>u(x)$ for all $x>z_{\Lambda}$. Then $u$ satisfies

$$
-u^{\prime \prime}=\Lambda^{-1} g_{\infty}(u) \quad \text { in }\left(z_{\Lambda}, \infty\right) .
$$

Noting $E[u, \Lambda](x) \equiv 0$ in $\left[z_{\Lambda}, \infty\right)$ and $u\left(z_{\Lambda}\right)=s_{\infty}$, we have

$$
\left(u^{\prime}\left(z_{\Lambda}\right), u\left(z_{\Lambda}\right)\right)=\left(u_{\Lambda, \alpha_{0}}^{\prime}\left(x_{\Lambda}\right), u_{\Lambda, \alpha_{0}}\left(x_{\Lambda}\right)\right)=\left(u_{1}^{\prime}\left(y_{1}\right), u_{1}\left(y_{1}\right)\right) .
$$


Thus it is easily seen from the construction of $u_{1}$ and the unique solvability of the initial value problem for (A.5) with $z=z_{\Lambda}$ that $u(x)=u_{1}\left(x+y_{1}-z_{\Lambda}\right)$ in R. Noting that

$$
u_{1}(0)=\max _{\mathbf{R}} u_{1}>u_{1}(x) \text { for } x \neq 0, \quad u(0)=\max _{\mathbf{R}} u,
$$

we deduce that $y_{1}=z_{\Lambda}$ and $u_{1} \equiv u$. Hence, the uniqueness of solutions of (2.1) holds. Similarly, we can prove the uniqueness of solutions of (2.2).

Remark that the above argument can be applied to conclude $u \equiv u_{1}$ if $u$ satisfies $-\mathcal{M}_{\lambda, \Lambda}^{ \pm}\left(u^{\prime \prime}\right)=g_{\infty}(u)$ in $\mathbf{R}$ with $u(0)=\max _{\mathbf{R}} u, u>0$ in $\mathbf{R}$ and $u(x) \rightarrow 0$ as either $x \rightarrow \infty$ or $x \rightarrow-\infty$. Thus we complete the proof.

\section{REFERENCES}

[1] S. Armstrong and B. Sirakov, Sharp Liouville results for fully nonlinear equations with power-growth nonlinearities. Ann. Sc. Norm. Super. Pisa Cl. Sci. (5) 10 (2011), no. $3,711-728.2$

[2] H. Berestycki and P.-L. Lions, Nonlinear scalar field equations. I. Existence of a ground state. Arch. Rational Mech. Anal. 82 (1983), no. 4, 313-345. 27

[3] A. Cutrí and F. Leoni, On the Liouville property for fully nonlinear equations. Ann. Inst. H. Poincaré Anal. Non Linéaire 17 (2000), no. 2, 219-245. 2

[4] D.G. de Figueiredo, P.-L. Lions and R.D. Nussbaum, A priori estimates and existence of positive solutions of semilinear elliptic equations. J. Math. Pures Appl. (9) 61(1982), no. 1, 41-63. 3, 8

[5] P. Felmer and A. Quaas, Critical exponents for the Pucci's extremal operators. C. R. Math. Acad. Sci. Paris 335 (2002), no. 11, 909-914. 2

[6] P. Felmer and A. Quaas, On critical exponents for the Pucci's extremal operators. Ann. Inst. H. Poincaré Anal. Non Linéaire 20 (2003), no. 5, 843-865. 2

[7] P. Felmer and A. Quaas, Positive radial solutions to a 'semilinear' equation involving the Pucci's operator. J. Differential Equations 199 (2004), no. 2, 376-393. 1, 3, 8

[8] G. Galise, F. Leoni and F. Pacella, Existence results for fully nonlinear equations in radial domains. Comm. Partial Differential Equations 42 (2017), no. 5, 757-779. 2

[9] L. Jeanjean and K. Tanaka, A note on a mountain pass characterization of least energy solutions. Adv. Nonlinear Stud. 3 (2003), no. 4, 445-455. 27

[10] M. Struwe, Variational methods. Applications to nonlinear partial differential equations and Hamiltonian systems. Fourth edition. Ergebnisse der Mathematik und ihrer Grenzgebiete. 3. Folge. A Series of Modern Surveys in Mathematics, 34. SpringerVerlag, Berlin, 2008. 1

[11] M. Willem, Minimax theorems. Progress in Nonlinear Differential Equations and their Applications, 24. Birkhäuser Boston, Inc., Boston, MA, 1996. 1

Patricio Felmer - Departamento de Ingeniería Matemática and CMM (UMi 2807 CNRS), Universidad de Chile, Casilla 170 Correo 3, Santiago, Chile pfelmer@dim.uchile.cl.

Norihisa Ikoma - Faculty of Mathematics and Physics, Institute of Science and Engineering, Kanazawa University, Kakuma, Kanazawa, Ishikawa 9201192, JAPAN

ikoma@se.kanazawa-u.ac.jp. 